\title{
Aminals as substrates for sulfur ylides: A synthesis of functionalised aziridines and $\mathrm{N}$-heterocycles
}

\author{
Christoforos G. Kokotos, and Varinder K. Aggarwal* \\ School of Chemistry, Bristol University, Cantock's Close, Bristol, UK BS8 1TS. \\ E-mail: v.aggarwal@bristol.ac.uk; Fax: +44 (0)117 929 8611; Tel: +44 (0)117 9546315
}

\section{Supplementary Information}

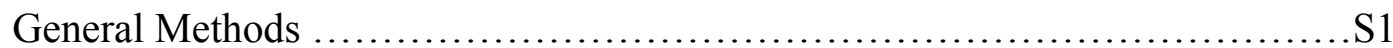

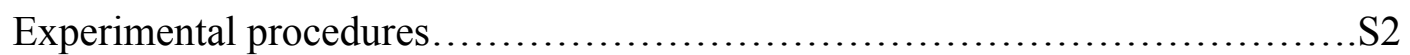

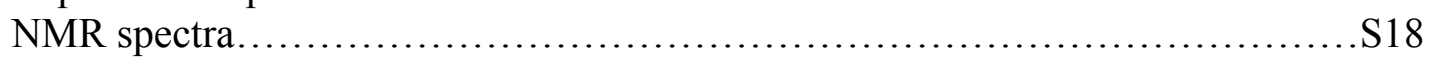

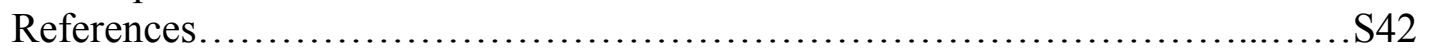

\section{General Methods}

All chemicals were purchased from Aldrich, Fluka or Lancaster. Anhydrous THF, $\mathrm{CH}_{2} \mathrm{Cl}_{2}$ were obtained from a purification column composed of activated alumina (A-2). Chromatography: Flash chromatography was performed on silica gel (Merck Kieselgel $60 \mathrm{~F}_{254}$ 230-400 mesh). TLC was performed on aluminum backed silica plates $\left(0.2 \mathrm{~mm}, 60 \mathrm{~F}_{254}\right)$ which were developed using standard visualising agents: UV fluorescence (254 \& $366 \mathrm{~nm}$ ), phosphomolybdic acid / $\Delta$, anisaldehyde / $\Delta$, potassium permanganate $/ \Delta$. Melting points were determined on a Khofler hot stage apparatus. Infra red spectra were recorded on a Perkin-Elmer Spectrum One FTIR spectrometer. Only selected absorbencies $\left(v_{\max }\right)$ are reported. ${ }^{1} \mathrm{H}$ NMR spectra were recorded at either 270 or $400 \mathrm{MHz}$ on Delta GX/270 or Delta GX/400 instruments respectively. Chemical shifts $\left(\delta_{\mathrm{H}}\right)$ are quoted in parts per million $(\mathrm{ppm})$, referenced to TMS. ${ }^{13} \mathrm{C}$ NMR spectra were recorded at either 68 or $100 \mathrm{MHz}$ on Delta GX/270 or Delta GX/400 instruments respectively. Chemical shifts $\left(\delta_{c}\right)$ are quoted in parts per million (ppm), referenced to the appropriate solvent peak and are assigned as $\mathrm{s}$, d, t, q for $\mathrm{C}, \mathrm{CH}, \mathrm{CH}_{2}, \mathrm{CH}_{3}$ respectively. Low resolution mass spectra $(\mathrm{m} / \mathrm{z})$ were recorded on a Micromass Analytical Autospec spectrometer, with only molecular ions $\left(\mathrm{M}^{+}\right.$or $\left.\mathrm{MH}^{+}\right)$and major peaks being reported with intensities quoted as percentages of the base peak. High-resolution mass spectra were recorded on a Micromass Analytical Autospec Spectrometer. All GC-MS experiments were performed using an Agilent 
6890 apparatus and the following conditions: column: HP190915-433 HP-5MS 5\% Phenyl Methyl Siloxane, capillary $30 \mathrm{~m}$ x $250 \mu \mathrm{m}$ x $0.25 \mu \mathrm{m}$ nominal, carrier gas: helium $1 \mathrm{~mL} / \mathrm{min}$ (constant flow mode), injector: $250{ }^{\circ} \mathrm{C}$ (split less mode), detector: agilent MSD 5973 (EI mode), Oven: $70{ }^{\circ} \mathrm{C}(3 \mathrm{~min}), 15^{\circ} \mathrm{C} / \mathrm{min}(15.3 \mathrm{~min}), 300{ }^{\circ} \mathrm{C}(8$ $\min )$.

\section{Experimental procedures}

\section{$N$-[(4-Methylphenyl)sulfonyl]pyrrolidin-2-ol}<smiles>OC1CCCN1[13I]</smiles>

For the synthesis see: Kokotos, C. G.; Aggarwal, V. K. Chem. Comm., 2006, 2156.

\section{tert-Butyl 2-hydroxy-1-pyrrolidinecarboxylate}<smiles>CC(C)(C)OC(=O)N1CCCC1O</smiles>

For the synthesis see: Dieter, R. K.; Sharma, R. R. J. Org. Chem., 1996, 61, 4180.

\section{General procedure for chiral imine synthesis}

To a stirred solution of $\mathrm{N}$-[(4-Methylphenyl)sulfonyl]pyrrolidin-2-ol or tert-Butyl 2hydroxy-1-pyrrolidinecarboxylate (1 eq.) in $\mathrm{CH}_{2} \mathrm{Cl}_{2}(15 \mathrm{~mL})$, $\mathrm{Ti}(\mathrm{OEt})_{4}(20 \%$ solution in ethanol) ( 2 eq.) was added under argon at room temperature. The solution was treated with $(R)$-2-methyl-2-propanesulfinamide (1.1 eq.) in one portion and the reaction mixture was then heated at reflux for $7 \mathrm{~h}$ under argon. The reaction mixture was allowed to cool to room temperature before quenching with an equal amount of brine $(15 \mathrm{~mL})$. The resulting slurry was then filtered through Celite, washed with an excess of $\mathrm{CH}_{2} \mathrm{Cl}_{2}(100 \mathrm{~mL})$ and the filtrate partitioned between brine $(80 \mathrm{~mL})$ and $\mathrm{CH}_{2} \mathrm{Cl}_{2}(80 \mathrm{~mL})$. The aqueous layer was extracted with $\mathrm{CH}_{2} \mathrm{Cl}_{2}(3 \times 75 \mathrm{~mL})$ and the 
combined organic layers were dried $\left(\mathrm{MgSO}_{4}\right)$ and concentrated under reduced pressure. The residue was then purified by flash chromatography eluting with 1:1 EtOAc:pet. ether followed by EtOAc to give the product.

\section{(R) $s^{-N 2-1-[(4-M e t h y l p h e n y l) s u l f o n y l] t e t r a h y d r o-1 H-2-p y r r o l y l-2-m e t h y l-2-~}$} propanesulfinamide $4^{1}$

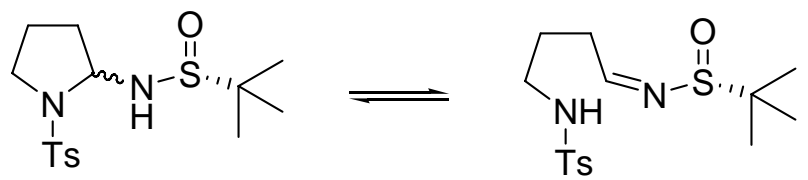

Colourless gum (81\%) as a mixture of ring opened and ring closed form; $R_{f}$ (EtOAc:pet.ether, 1:1) 0.1/0.15/0.3; IR (film) 2957 (NH), $2925(\mathrm{NH}), 2870(\mathrm{NH})$, $1597(\mathrm{HC}=\mathrm{N}), 1335(\mathrm{~S}=\mathrm{O}), 1155\left(\mathrm{SO}_{2}\right), 1091(\mathrm{~S}=\mathrm{O}), 1033(\mathrm{~S}=\mathrm{O}) \mathrm{cm}^{-1}$; open form $\delta_{\mathrm{H}}$ $\left(400 \mathrm{MHz}, \mathrm{CDCl}_{3}\right) 8.01(1 \mathrm{H}, \mathrm{t}, J 4.0 \mathrm{~Hz}, \mathrm{~N}=\mathrm{CH}), 7.72(2 \mathrm{H}, \mathrm{d}, J 8.3 \mathrm{~Hz}, \mathrm{Ar}), 7.29$ $(2 \mathrm{H}, \mathrm{d}, J 8.3 \mathrm{~Hz}, \mathrm{ArH}), 4.74(1 \mathrm{H}, \mathrm{t}, J 6.3 \mathrm{~Hz}, \mathrm{NH}), 3.04-2.97\left(2 \mathrm{H}, \mathrm{m}, \mathrm{NCH}_{2}\right), 2.53$ $\left(2 \mathrm{H}, \mathrm{td}, J 7.1\right.$ and $\left.4.0 \mathrm{~Hz}, \mathrm{CH}_{2} \mathrm{C}=\mathrm{N}\right), 2.42\left(3 \mathrm{H}, \mathrm{s}, \mathrm{CH}_{3}\right) 1.81\left(2 \mathrm{H}, \mathrm{m}, \mathrm{NCH}_{2} \mathrm{CH}_{2}\right), 1.15$ $\left[9 \mathrm{H}, \mathrm{s}, \mathrm{C}\left(\mathrm{CH}_{3}\right)_{3}\right]$; closed form two diastereomers $(1: 1) \delta_{\mathrm{H}}\left(400 \mathrm{MHz}, \mathrm{CDCl}_{3}\right) 7.80-$ $7.70(4 \mathrm{H}, \mathrm{d}, J 8.3 \mathrm{~Hz}, \mathrm{Ar}), 7.33-7.27(4 \mathrm{H}, \mathrm{d}, J 8.3 \mathrm{~Hz}, \mathrm{ArH}), 5.18(1 \mathrm{H}, \mathrm{td}, J 6.8$ and $2.6 \mathrm{~Hz}, \mathrm{NCH}), 5.05(1 \mathrm{H}, \mathrm{dt}, J 6.3$ and $2.4 \mathrm{~Hz}, \mathrm{NCH}), 4.16(1 \mathrm{H}, \mathrm{d}, J 2.4 \mathrm{~Hz}, \mathrm{NH}), 3.89$ $(1 \mathrm{H}, \mathrm{d}, J 6.8 \mathrm{~Hz}, \mathrm{NH}), 3.59(1 \mathrm{H}, \mathrm{ddd}, J 10.3,7.5$ and $3.1 \mathrm{~Hz}, \mathrm{NCHH}), 3.42(1 \mathrm{H}$, ddd, $J$ 11.3, 6.2 and $2.5 \mathrm{~Hz}, \mathrm{NCHH}), 3.15-3.07(2 \mathrm{H}, \mathrm{m}, 2 \mathrm{x} \mathrm{NCHH}), 2.72-2.55(4 \mathrm{H}, \mathrm{m}, 2 \mathrm{x}$ $\left.\mathrm{CH}_{2} \mathrm{CHN}\right), 2.41\left(6 \mathrm{H}, \mathrm{s}, 2 \times \mathrm{CH}_{3}\right) 2.20-1.61\left(4 \mathrm{H}, \mathrm{m}, 2 \times \mathrm{CH}_{2} \mathrm{CH}_{2}\right), 1.23[9 \mathrm{H}, \mathrm{s}$, $\left.\mathrm{C}\left(\mathrm{CH}_{3}\right)_{3}\right], 1.23\left[9 \mathrm{H}, \mathrm{s}, \mathrm{C}\left(\mathrm{CH}_{3}\right)_{3}\right]$; ring-open and ring closed forms $\delta_{\mathrm{c}}(100.5 \mathrm{MHz}$, $\left.\mathrm{CDCl}_{3}\right) 168.4$ (d), 144.0 (s), 143.8 (s), 143.3 (s), 137.3 (s), 137.2 (s), 137.1 (s), 130.0 (d), 129.8 (d), 129.7 (d), 127.7 (d), 127.5 (d), 127.1 (d), 72.8 (d), 70.6 (d), 56.7 (s), $56.3(\mathrm{~s}), 56.0(\mathrm{~s}), 48.6(\mathrm{t}), 48.1(\mathrm{t}), 42.6(\mathrm{t}), 34.1(\mathrm{t}), 33.2(\mathrm{t}), 32.2(\mathrm{t}), 25.3(\mathrm{t}), 23.6(\mathrm{t})$, 23.2 (t), 22.6 (q), 22.5 (q), 22.4 (q), 21.6 (q), 21.5 (q), 21.1 (q); MS (ESI) $m / z$ (\%) 367 $\left(\mathrm{M}+\mathrm{Na}^{+}, 25 \%\right)$ and $345\left(\mathrm{MH}^{+}, 23 \%\right)$; HRMS (ESI) found 367.1121. $\mathrm{C}_{15} \mathrm{H}_{24} \mathrm{~N}_{2} \mathrm{O}_{3} \mathrm{~S}_{2} \mathrm{Na}$ requires 367.1115 . 
$(R)_{s}$-2-(2-Methyl-propane-2-sulfinylamino)-pyrrolidine-1-carboxylic acid tertbutyl ester

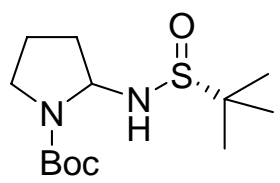

Colourless oil (39\%); $\mathrm{R}_{\mathrm{f}}$ (EtOAc:pet.ether, 1:1) 0.05; IR (film) $2952(\mathrm{NH})$, $1641(\mathrm{OCONH}), 1335(\mathrm{~S}=\mathrm{O}) \mathrm{cm}^{-1} ; \delta_{\mathrm{H}}\left(400 \mathrm{MHz}, \mathrm{CDCl}_{3}\right)$ 5.20-5.16 $(1 \mathrm{H}, \mathrm{m}, \mathrm{NCH})$, 3.80-3.78 (1H, m, NH), 3.49-3.47 (1H, m, NCHH), 3.29-3.25 (1H, m, NCHH), 2.10$1.80\left(4 \mathrm{H}, \mathrm{m}, \mathrm{CH}_{2} \mathrm{CH}_{2}\right), 1.44\left[9 \mathrm{H}, \mathrm{s}, \mathrm{C}\left(\mathrm{CH}_{3}\right)_{3}\right], 1.18\left[9 \mathrm{H}, \mathrm{s}, \mathrm{C}\left(\mathrm{CH}_{3}\right)_{3}\right] ; \delta_{\mathrm{c}}(100.5 \mathrm{MHz}$, $\left.\mathrm{CDCl}_{3}\right) 154.7$ (s), 83.2 (d), 79.9 (s), 56.7 (s), 45.2 (t), 28.4 (q), 28.3 (t), 22.4 (q), 22.3 (t). MS (CI) $m / z(\%) 291\left(\mathrm{MH}^{+}, 100 \%\right)$ and $186\left(\mathrm{MH}^{+}-\mathrm{SOBu}^{\mathrm{t}}, 45 \%\right)$; (Found: $\mathrm{C}$, $53.92 \% ; \mathrm{H}, 8.89 \% ; \mathrm{N}, 9.43 \% . \mathrm{C}_{13} \mathrm{H}_{26} \mathrm{~N}_{2} \mathrm{O}_{3} \mathrm{~S}$ requires $\mathrm{C}, 53.76 \% ; \mathrm{H}, 9.02 \% ; \mathrm{N}$, $9.65 \%) ;[\alpha]_{\mathrm{D}}^{23}-104\left(c 1.0, \mathrm{CH}_{2} \mathrm{Cl}_{2}\right)$.

\section{Reaction of aminal 4 with achiral sulfonium salt 5}

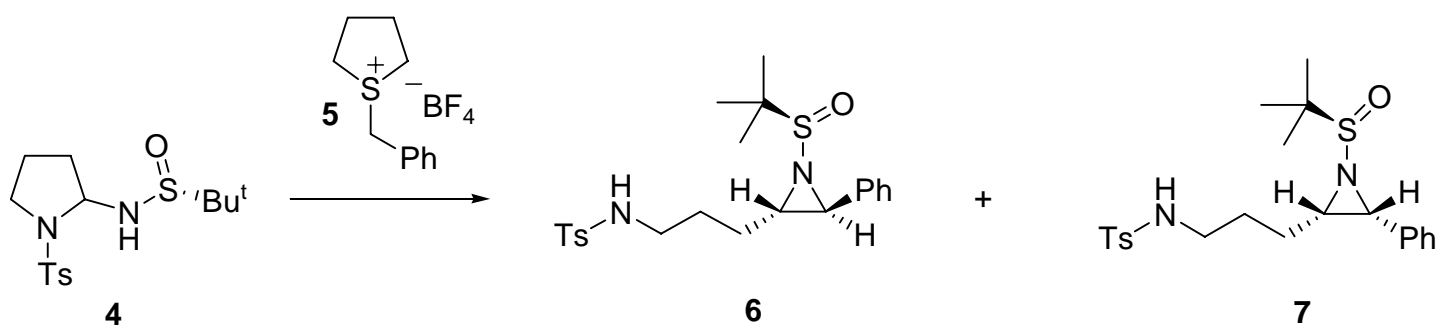

To a solution of N2-1-[(4-methylphenyl)sulfonyl]tetrahydro-1H-2-pyrrolyl-2methyl-2-propanesulfinamide $4(0.10 \mathrm{~g}, 0.29 \mathrm{mmol})$ in THF $(4 \mathrm{~mL})$ at $0{ }^{\circ} \mathrm{C}$ was added $\mathrm{P}_{2}$ base $(0.10 \mathrm{~mL}, 0.3 \mathrm{mmol})$ and the reaction mixture was left stirring for $30 \mathrm{~min}$. 1Benzyltetrahydrothiophenium tetrafluoroborate $5(0.12 \mathrm{~g}, 0.44 \mathrm{mmol})$ was added at 0 ${ }^{\circ} \mathrm{C}$ followed by $\mathrm{P}_{2}$ base $(0.10 \mathrm{~mL}, 0.3 \mathrm{mmol})$. The reaction mixture was then stirred for 3.5 hours at $0{ }^{\circ} \mathrm{C}$, diluted with $\mathrm{CH}_{2} \mathrm{Cl}_{2}(20 \mathrm{~mL})$ and $\mathrm{H}_{2} \mathrm{O}(20 \mathrm{~mL})$. The aqueous layer was extracted with $\mathrm{CH}_{2} \mathrm{Cl}_{2}(3 \times 20 \mathrm{~mL})$. The combined organic layers were dried $\left(\mathrm{MgSO}_{4}\right)$. The solvents were removed in vacuo and the resultant residue was purified by column chromatography, eluting with 1:1 pet. ether:EtOAc to give: 
$(R)_{\underline{s}}-(2 S)-(3 R)-4-m e t h y l-N-\{3-[3-p h e n y l-1-($ propane-2-sulfinyl)-aziridin-2-yl]propyl -benzenesulfonamide (6) (major trans) as a colourless oil (63 $\mathrm{mg}, 50 \%) ; R_{\mathrm{f}}$ (EtOAc:pet. ether, 1:1) 0.50; IR (film) 3063 (NH), 1599 (Ar), $1338\left(\mathrm{SO}_{2}\right), 1159$ $\left(\mathrm{SO}_{2}\right), 1058(\mathrm{SO}) \mathrm{cm}^{-1} ; \delta_{\mathrm{H}}\left(400 \mathrm{MHz}, \mathrm{CDCl}_{3}\right) 7.72(2 \mathrm{H}, \mathrm{d}, J 8.3 \mathrm{~Hz}, \mathrm{ArH}), 7.31-7.25$ (5H, m, ArH), 7.19 (2H, d, J 8.3 Hz, ArH), $5.24(1 \mathrm{H}, \mathrm{t}, J 6.1 \mathrm{~Hz}, \mathrm{NH}), 3.11(1 \mathrm{H}, \mathrm{d}, J$ $3.9 \mathrm{~Hz}, \mathrm{NCHPh}), 3.05-2.91\left(2 \mathrm{H}, \mathrm{m}, \mathrm{NCH}_{2}\right), 2.51(1 \mathrm{H}, \mathrm{td}, J 6.4$ and $3.9 \mathrm{~Hz}$, $\mathrm{CHNCHPh}), 2.41\left(3 \mathrm{H}, \mathrm{s}, \mathrm{CH}_{3}\right), 2.21-2.13\left(1 \mathrm{H}, \mathrm{m}, \mathrm{CHHCH}_{2}\right), 2.04-1.96(1 \mathrm{H}, \mathrm{m}$, $\mathrm{CHHCHH}), 1.79-1.63(2 \mathrm{H}, \mathrm{m}, \mathrm{CH} H \mathrm{H} H \mathrm{H}), 1.19\left[9 \mathrm{H}, \mathrm{s}, \mathrm{C}\left(\mathrm{CH}_{3}\right)_{3}\right] ; \delta_{\mathrm{c}}(100.5 \mathrm{MHz}$, $\left.\mathrm{CDCl}_{3}\right) 143.3$ (s), 143.2 (s), 136.8 (s), 129.7 (d), 128.7 (d), 128.0 (d), 127.2 (d), 126.3 (d), $57.1(\mathrm{~s}), 50.2(\mathrm{~d}), 46.1$ (d), $42.5(\mathrm{t}), 28.2(\mathrm{t}), 25.8(\mathrm{t}), 22.3$ (q), $21.4(\mathrm{q}) ; \mathrm{MS}(\mathrm{CI})$ : $m / z(\%) 435\left(\mathrm{MH}^{+}, 87 \%\right), 378\left(\mathrm{MH}^{+}-\mathrm{Bu}^{\mathrm{t}}, 17 \%\right)$ and $224\left(\mathrm{M}^{+}-\mathrm{NSOBu}^{\mathrm{t}}-\mathrm{CH}_{2} \mathrm{Ph}, 100 \%\right)$; HRMS (CI) found 435.1776. $\mathrm{C}_{22} \mathrm{H}_{30} \mathrm{~N}_{2} \mathrm{O}_{3} \mathrm{~S}_{2}$ requires 435.1772 (Found: $\mathrm{C}, 61.02 \%$; $\mathrm{H}$, $6.73 \% ; \mathrm{N}, 6.21 \% . \mathrm{C}_{22} \mathrm{H}_{30} \mathrm{~N}_{2} \mathrm{O}_{3} \mathrm{~S}_{2}$ requires $\left.\mathrm{C}, 60.80 \% ; \mathrm{H}, 6.96 \% ; \mathrm{N}, 6.45 \%\right) ;[\alpha]_{\mathrm{D}}{ }^{23}-36$ (c $1.0, \mathrm{CH}_{2} \mathrm{Cl}_{2}$ ).

4-methyl- $N$ - $\{3$-[3-phenyl-1-(propane-2-sulfinyl)-aziridin-2-yl]-propyl $\}$ -

benzenesulfonamide (6 minor:7) (minor trans:cis 5:1) as a colourless oil (32 $\mathrm{mg}$, 27\%); $R_{\mathrm{f}}$ (EtOAc:pet. ether, 1:1) 0.55; IR (film) $3063(\mathrm{NCH}), 1599$ (Ar), $1330\left(\mathrm{SO}_{2}\right)$, $1158\left(\mathrm{SO}_{2}\right), 1093(\mathrm{SO}) \mathrm{cm}^{-1}$; minor trans $\delta_{\mathrm{H}}\left(400 \mathrm{MHz}, \mathrm{CDCl}_{3}\right) 7.72(2 \mathrm{H}, \mathrm{d}, J 8.3 \mathrm{~Hz}$, ArH), 7.33-7.24 (7H, m, ArH), $4.87(1 \mathrm{H}, \mathrm{t}, J 6.2 \mathrm{~Hz}, \mathrm{NH}), 3.33(1 \mathrm{H}, \mathrm{d}, J 3.9 \mathrm{~Hz}$, $\mathrm{NCHPh}), 2.98\left(2 \mathrm{H}, \mathrm{q}, J 6.2 \mathrm{~Hz}, \mathrm{NCH}_{2}\right), 2.43-2.34\left(4 \mathrm{H}, \mathrm{m}, \mathrm{CH}_{3}\right.$ and $\mathrm{CHNCHPh}$ ), 1.96-1.83 (1H, m, $\mathrm{CHHCH} 2), 1.82-1.60(3 \mathrm{H}, \mathrm{m}, \mathrm{CHHCHH}), 1.12\left[9 \mathrm{H}, \mathrm{s}, \mathrm{C}\left(\mathrm{CH}_{3}\right)_{3}\right] ; \delta_{\mathrm{c}}$ (100.5 MHz, $\left.\mathrm{CDCl}_{3}\right) 143.4$ (s), 143.2 (s), 137.6 (s), 129.8 (d), 128.0 (d), 128.6 (d), $127.2(\mathrm{~d}), 127.1$ (d), 57.4 (s), 50.9 (d), 47.4 (d), $42.8(\mathrm{t}), 28.3(\mathrm{t}), 25.9$ (t), $22.8(\mathrm{q})$, 21.5 (q); $\underline{c i s} \delta_{\mathrm{H}}\left(400 \mathrm{MHz}, \mathrm{CDCl}_{3}\right) 7.70(2 \mathrm{H}, \mathrm{d}, J 8.3 \mathrm{~Hz}, \mathrm{ArH}), 7.33-7.24(5 \mathrm{H}, \mathrm{m}$, ArH), 7.17 (2H, d, J 8.3 Hz, ArH), 4.41 (1H, t, J 6.3 Hz, NH), 3.79 (1H, d, J 7.0 Hz, $\mathrm{NCHPh}), 2.88-2.77\left(2 \mathrm{H}, \mathrm{m}, \mathrm{NCH}_{2}\right), 2.43-2.34\left(4 \mathrm{H}, \mathrm{m}, \mathrm{CH}_{3}\right.$ and $\left.\mathrm{CHNCHPh}\right), 1.96-$ $1.83\left(1 \mathrm{H}, \mathrm{m}, \mathrm{C} H \mathrm{HCH}_{2}\right), 1.82-1.60(3 \mathrm{H}, \mathrm{m}, \mathrm{CH} \mathrm{CHH}), 1.17\left[9 \mathrm{H}, \mathrm{s}, \mathrm{C}\left(\mathrm{CH}_{3}\right)_{3}\right] ; \delta_{\mathrm{c}}$ (100.5 MHz, $\left.\mathrm{CDCl}_{3}\right) 143.6$ (s), 143.4 (s), 137.3 (s), 129.7 (d), 128.4 (d), 128.1 (d), $127.6(\mathrm{~d}), 126.6(\mathrm{~d}), 57.0(\mathrm{~s}), 48.0(\mathrm{~d}), 46.7(\mathrm{~d}), 42.5(\mathrm{t}), 28.2(\mathrm{t}), 24.8(\mathrm{t}), 22.7(\mathrm{q})$, 21.5 (q); MS (CI): $m / z(\%) 435\left(\mathrm{MH}^{+}, 67 \%\right), 330\left(\mathrm{MH}^{+}-\mathrm{SOBu}^{\mathrm{t}}, 15 \%\right)$ and $224\left(\mathrm{M}^{+}-\right.$ $\left.\mathrm{NSOBu}^{\mathrm{t}}-\mathrm{CH}_{2} \mathrm{Ph}, 100 \%\right)$. 


\section{Reaction of aminal 4 with achiral sulfonium salt 5 leading to pyrrolidines and piperidine}

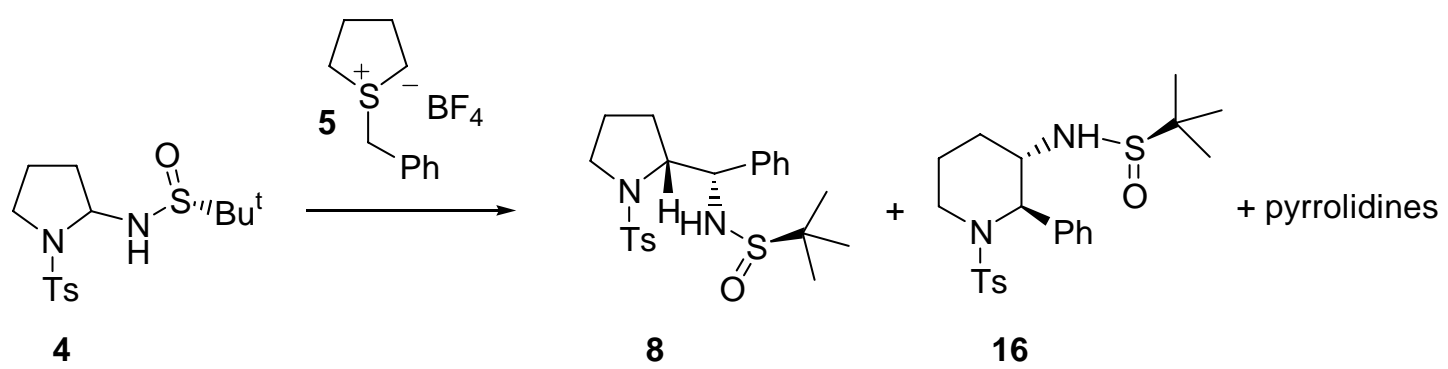

To a solution of N2-1-[(4-methylphenyl)sulfonyl]tetrahydro-1H-2-pyrrolyl-2methyl-2-propanesulfinamide $4(0.10 \mathrm{~g}, 0.29 \mathrm{mmol})$ in THF $(4 \mathrm{~mL})$ at $0{ }^{\circ} \mathrm{C}$ was added $\mathrm{P}_{2}$ base $(0.10 \mathrm{~mL}, 0.3 \mathrm{mmol})$ and the reaction mixture was left stirring for $30 \mathrm{~min} .1$ Benzyltetrahydrothiophenium tetrafluoroborate $5(0.12 \mathrm{~g}, 0.44 \mathrm{mmol})$ was added at 0 ${ }^{\circ} \mathrm{C}$ followed by $\mathrm{P}_{2}$ base $(0.10 \mathrm{~mL}, 0.3 \mathrm{mmol})$. The reaction mixture was stirred for 5 hours at $0{ }^{\circ} \mathrm{C}$ and then heated to reflux for $15 \mathrm{~h}$. The reaction was diluted with $\mathrm{CH}_{2} \mathrm{Cl}_{2}$ (20 mL) and $\mathrm{H}_{2} \mathrm{O}(20 \mathrm{~mL})$. The aqueous layer was extracted with $\mathrm{CH}_{2} \mathrm{Cl}_{2}(3 \times 20$ $\mathrm{mL})$. The combined organic layers were dried $\left(\mathrm{MgSO}_{4}\right)$. The solvents were removed in vacuo and the resultant residue was purified by column chromatography, eluting with 1:1 pet. ether:EtOAc to give:

$(R)_{\underline{s}}-(R)-\alpha-(2 R)-2-M e t h y l p r o p a n e-2-s u l f i n i c$ acid \{phenyl-[1-(toluene-4-sulfonyl)pyrrolodin-2-yl]-methyl \}amide (8) (major) as a white solid (58 $\mathrm{mg}, 46 \%$ ) $\mathrm{mp}$ 72-75 ${ }^{\circ} \mathrm{C}$ (pet. ether); $R_{\mathrm{f}}$ (EtOAc:pet. ether, 1:1) 0.30; IR (film) 1599 (Ar), $1341\left(\mathrm{SO}_{2}\right), 1159$ $\left(\mathrm{SO}_{2}\right), 1058(\mathrm{SO}) \mathrm{cm}^{-1} ; \delta_{\mathrm{H}}\left(400 \mathrm{MHz}, \mathrm{CDCl}_{3}\right) 7.78(2 \mathrm{H}, \mathrm{d}, J 8.3 \mathrm{~Hz}, \mathrm{ArH}), 7.43-$ $7.21(7 \mathrm{H}, \mathrm{m}, \mathrm{ArH}), 5.55(1 \mathrm{H}, \mathrm{d}, J 3.5 \mathrm{~Hz}, \mathrm{NH}), 4.44$ [1H, t (ap), J $3.5 \mathrm{~Hz}, \mathrm{NCHPh}$ ], $4.20(1 \mathrm{H}, \mathrm{m}, \mathrm{NCHCHPh}), 3.09(1 \mathrm{H}, \mathrm{dt}, J 10.9$ and $7.4 \mathrm{~Hz}, \mathrm{NCHH}), 2.80(1 \mathrm{H}, \mathrm{ddd}, J$ 10.9, 7.4 and $5.7 \mathrm{~Hz}, \mathrm{NCHH}), 2.41\left(3 \mathrm{H}, \mathrm{s}, \mathrm{CH}_{3}\right), 1.79-1.71\left(1 \mathrm{H}, \mathrm{m}, \mathrm{CH} \mathrm{HCH}_{2}\right), 1.63-$ $1.58(1 \mathrm{H}, \mathrm{m}, \mathrm{CHHCHH}), 1.56-1.42(2 \mathrm{H}, \mathrm{m}, \mathrm{CHHCHH}), 1.24\left[9 \mathrm{H}, \mathrm{s}, \mathrm{C}\left(\mathrm{CH}_{3}\right)_{3}\right] ; \delta_{\mathrm{c}}$ (100.5 MHz, $\left.\mathrm{CDCl}_{3}\right) 143.9$ (s), 138.8 (s), 134.1 (s), 129.7 (d), 128.6 (d), 128.0 (d), $127.3(\mathrm{~d}), 127.0$ (d), 65.0 (d), 61.8 (d), 55.9 (s), $50.1(\mathrm{t}), 29.1$ (t), $22.8(\mathrm{t}), 22.7$ (q), 21.5 (q); MS (CI): $m / z(\%) 435\left(\mathrm{MH}^{+}, 85 \%\right)$ and $224\left(\mathrm{M}^{+}-\mathrm{NSOBu}^{\mathrm{t}}-\mathrm{CH}_{2} \mathrm{Ph}, 100 \%\right)$; HRMS (CI) found 435.1776. $\mathrm{C}_{22} \mathrm{H}_{30} \mathrm{~N}_{2} \mathrm{O}_{3} \mathrm{~S}_{2}$ requires 435.1772; $[\alpha]_{\mathrm{D}}{ }^{23}-76$ (c 1.0, $\mathrm{CH}_{2} \mathrm{Cl}_{2}$ ). 
piperidin-3-yl]-amide (16) as a white solid (15 mg, $12 \%) \mathrm{mp} 79-82{ }^{\circ} \mathrm{C}$ (pet. ether); $R_{\mathrm{f}}$ (EtOAc:pet. ether, 1:1) 0.25; IR (film) 1599 (Ar), $1326\left(\mathrm{SO}_{2}\right), 1159\left(\mathrm{SO}_{2}\right), 1058$ (SO) $\mathrm{cm}^{-1}$; $\delta_{\mathrm{H}}\left(400 \mathrm{MHz}, \mathrm{CDCl}_{3}\right) 7.69(2 \mathrm{H}, \mathrm{d}, J 8.3 \mathrm{~Hz}, \mathrm{ArH}), 7.30-7.19$ (5H, m, ArH), 7.04 (2H, d, J 8.3 Hz, ArH), 5.21 (1H, br s, NCHPh), 4.17 (1H, d, J 8.2 Hz, NH), 3.84 $(1 \mathrm{H}, \mathrm{ddd}, J$ 9.8, 8.2 and $2.0 \mathrm{~Hz}, \mathrm{NHCHCHPh}), 3.05(1 \mathrm{H}, \mathrm{td}, J 13.1$ and $2.5 \mathrm{~Hz}$, $\mathrm{NCHH}), 2.87(1 \mathrm{H}, \mathrm{dt}, J 13.1$ and $6.9 \mathrm{~Hz}, \mathrm{NCHH}), 2.41\left(3 \mathrm{H}, \mathrm{s}, \mathrm{CH}_{3}\right), 1.92-1.75(3 \mathrm{H}$, m, $\left.\mathrm{CHHCH} \mathrm{H}_{2}\right), 1.64-1.52(1 \mathrm{H}, \mathrm{m}, \mathrm{CHHCHH}), 1.24\left[9 \mathrm{H}, \mathrm{s}, \mathrm{C}\left(\mathrm{CH}_{3}\right)_{3}\right] ; \delta_{\mathrm{c}}(100.5 \mathrm{MHz}$, $\left.\mathrm{CDCl}_{3}\right) 143.3$ (s), 138.2 (s), 136.5 (s), 129.8 (d), 128.8 (d), 127.4 (d), 127.0 (d), 126.8 (d), $61.2(\mathrm{~d}), 59.1$ (d), $56.0(\mathrm{~s}), 50.6(\mathrm{t}), 24.3(\mathrm{t}), 23.8(\mathrm{t}), 22.8(\mathrm{q}), 21.5(\mathrm{q}) ; \mathrm{MS}(\mathrm{CI})$ : $m / z(\%) 435\left(\mathrm{MH}^{+}, 89 \%\right)$ and $224\left(\mathrm{M}^{+}-\mathrm{NSOBu}^{\mathrm{t}}-\mathrm{CH}_{2} \mathrm{Ph}, 100 \%\right)$; HRMS (CI) found 435.1776. $\mathrm{C}_{22} \mathrm{H}_{30} \mathrm{~N}_{2} \mathrm{O}_{3} \mathrm{~S}_{2}$ requires 435.1769; $[\alpha]_{\mathrm{D}}^{23}-32\left(c 1.0, \mathrm{CH}_{2} \mathrm{Cl}_{2}\right)$.

\section{Reaction of Boc aminal 4' with achiral sulfonium salt 5}

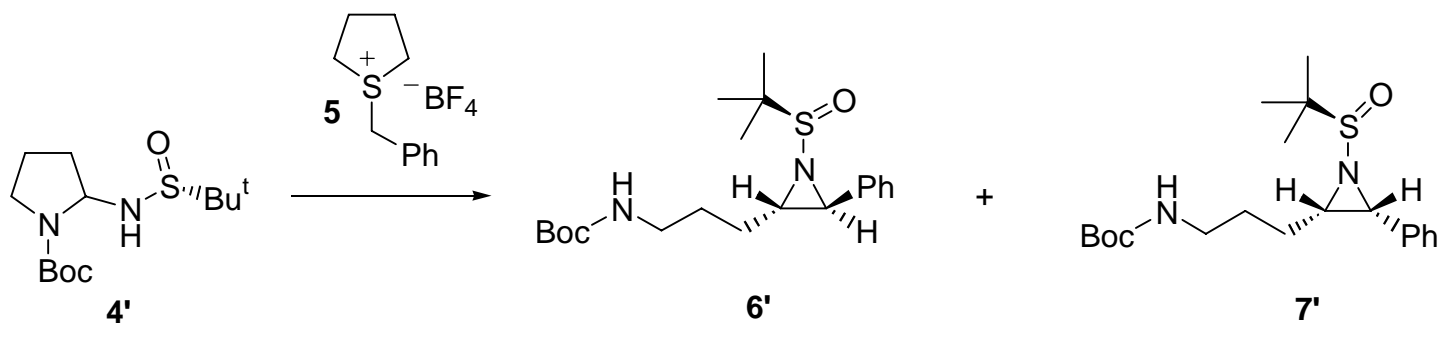

To a solution of 2-(2-methylpropane-2-sulfinylamino)-pyrrolidine-1carboxylic acid tert-butyl ester 4' $(0.10 \mathrm{~g}, 0.29 \mathrm{mmol})$ in THF $(4 \mathrm{~mL})$ at $0{ }^{\circ} \mathrm{C}$ was added $\mathrm{P}_{2}$ base $(0.10 \mathrm{~mL}, 0.3 \mathrm{mmol})$ and the reaction mixture was left stirring for 30 min. 1-Benzyltetrahydrothiophenium tetrafluoroborate $5(0.12 \mathrm{~g}, 0.44 \mathrm{mmol})$ was added at $0{ }^{\circ} \mathrm{C}$ followed by $\mathrm{P}_{2}$ base $(0.10 \mathrm{~mL}, 0.3 \mathrm{mmol})$. The reaction mixture was stirred for 3.5 hours at $0{ }^{\circ} \mathrm{C}$, diluted with $\mathrm{CH}_{2} \mathrm{Cl}_{2}(20 \mathrm{~mL})$ and $\mathrm{H}_{2} \mathrm{O}(20 \mathrm{~mL})$. The aqueous layer was extracted with $\mathrm{CH}_{2} \mathrm{Cl}_{2}(3 \times 20 \mathrm{~mL})$. The combined organic layers were washed with brine $(20 \mathrm{~mL})$, and dried $\left(\mathrm{MgSO}_{4}\right)$. The solvents were removed in vacuo and the resultant residue was purified by column chromatography, eluting with 1:1 pet. ether:EtOAc to give:

\{3-[1-(2-Methylpropane-2-sulfinyl)-3-phenylaziridin-2-yl]-propyl\}-carbamic acid tert-butyl ester (6’:7') (trans:cis) as a colourless oil (15:1) (23 mg, 39\%); $R_{\mathrm{f}}$ (EtOAc:pet. ether, 1:1) 0.25; IR (film) 3350 (NH), 2926 (Me), 1693 (OCONH), 1056 
(SO) $\mathrm{cm}^{-1}$; trans $\delta_{\mathrm{H}}\left(400 \mathrm{MHz}, \mathrm{CDCl}_{3}\right) 7.38-7.21(5 \mathrm{H}, \mathrm{m}, \mathrm{ArH}), 4.61(1 \mathrm{H}$, br s, NH), $3.42(1 \mathrm{H}, \mathrm{d}, J 3.9 \mathrm{~Hz}, \mathrm{NCHPh}), 3.22-3.12\left(2 \mathrm{H}, \mathrm{m}, \mathrm{NCH}_{2}\right), 2.97-2.93(1 \mathrm{H}, \mathrm{br} \mathrm{m}$, $\mathrm{CHNCHPh}), 2.00-1.95\left(1 \mathrm{H}, \mathrm{m}, \mathrm{CHHCH}_{2}\right), 1.83-1.62(3 \mathrm{H}, \mathrm{m}, \mathrm{CH} H \mathrm{CHH}), 1.43[9 \mathrm{H}$, s, $\left.\mathrm{C}\left(\mathrm{CH}_{3}\right)_{3}\right], 1.11\left[9 \mathrm{H}, \mathrm{s}, \mathrm{C}\left(\mathrm{CH}_{3}\right)_{3}\right] ; \delta_{\mathrm{c}}\left(100.5 \mathrm{MHz}, \mathrm{CDCl}_{3}\right) 154.3(\mathrm{~s}), 132.5(\mathrm{~s}), 129.0$ (d), 128.7 (d), 125.6 (d), 82.3 (s), 58.6 (s), 48.5 (d), 44.7 (d), 40.5 (t), 31.0 (t), 28.5 (q), 24.8 (t), 22.8 (q); $\underline{c i s} \delta_{\mathrm{H}}\left(400 \mathrm{MHz}, \mathrm{CDCl}_{3}\right)$ 7.38-7.21 (5H, m, ArH), $4.32(1 \mathrm{H}, \mathrm{br}$ s, NH), $3.84(1 \mathrm{H}, \mathrm{d}, J 7.0 \mathrm{~Hz}, \mathrm{NCHPh}), 3.12-2.98\left(2 \mathrm{H}, \mathrm{m}, \mathrm{NCH}_{2}\right), 2.47-2.41(1 \mathrm{H}, \mathrm{br}$ m, CHNCHPh), 2.00-1.95 (1H, m, $\left.\mathrm{CHHCH}_{2}\right), 1.83-1.62(3 \mathrm{H}, \mathrm{m}, \mathrm{CHHCHH}), 1.45$ [9H, s, $\left.\mathrm{C}\left(\mathrm{CH}_{3}\right)_{3}\right], 1.19\left[9 \mathrm{H}, \mathrm{s}, \mathrm{C}\left(\mathrm{CH}_{3}\right)_{3}\right] ; \delta_{\mathrm{c}}\left(100.5 \mathrm{MHz}, \mathrm{CDCl}_{3}\right) 154.3(\mathrm{~s}), 132.5$ (s), 129.0 (d), 128.7 (d), 125.6 (d), 82.4 (s), 57.4 (s), 40.5 (t), 38.7 (d), 36.5 (d), 31.0 (t), 28.5 (q), 24.8 (t), 22.8 (q); MS (CI): $m / z$ (\%) $381\left(\mathrm{MH}^{+}, 100\right)$; HRMS (CI) found 381.2212. $\mathrm{C}_{20} \mathrm{H}_{33} \mathrm{~N}_{2} \mathrm{O}_{3} \mathrm{~S}$ requires 381.2210..

\section{Reaction of aminal 4 with achiral sulfonium salt 9}

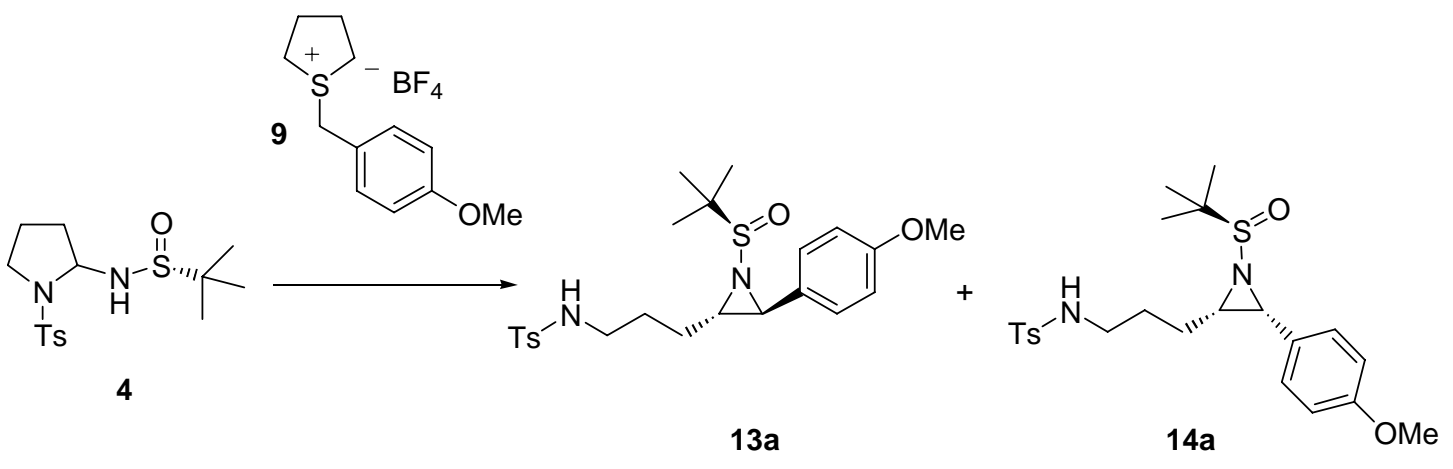

To a solution of N2-1-[(4-methylphenyl)sulfonyl]tetrahydro-1H-2-pyrrolyl-2-methyl2-propanesulfinamide $4(0.10 \mathrm{~g}, 0.29 \mathrm{mmol})$ in THF $(4 \mathrm{~mL})$ at $0{ }^{\circ} \mathrm{C}$ was added $\mathrm{P}_{2}$ base $(0.10 \mathrm{~mL}, 0.3 \mathrm{mmol})$ and the reaction mixture was left stirring for $30 \mathrm{~min}$. 1-4Methoxybenzyltetrahydrothiophenium tetrafluoroborate $9(0.13 \mathrm{~g}, 0.44 \mathrm{mmol})$ was added at $0{ }^{\circ} \mathrm{C}$ followed by $\mathrm{P}_{2}$ base $(0.10 \mathrm{~mL}, 0.3 \mathrm{mmol})$. The reaction mixture was then stirred for 3.5 hours at $0{ }^{\circ} \mathrm{C}$, diluted with $\mathrm{CH}_{2} \mathrm{Cl}_{2}(20 \mathrm{~mL})$ and $\mathrm{H}_{2} \mathrm{O}(20 \mathrm{~mL})$. The aqueous layer was extracted with $\mathrm{CH}_{2} \mathrm{Cl}_{2}(3 \times 20 \mathrm{~mL})$. The combined organic layers were washed with brine $(20 \mathrm{~mL})$, and dried $\left(\mathrm{MgSO}_{4}\right)$. The solvents were removed in vacuo and the resultant residue was purified by column chromatography, eluting with 1:1 pet. ether:EtOAc to give: 
$\underline{N \text {-\{3-[3-(4-Methoxyphenyl)-1-(2-methylpropane-2-sulfinyl)-aziridin-2-yl]-propyl\}- }}$ benzenesulfonamide (13a major:13a minor:14a) (major trans: minor trans:cis) as a colourless oil (2.9:1.6:1) (58 mg, 43\%); $R_{\mathrm{f}}$ (EtOAc:pet. ether, 1:1) 0.45; IR (film), 2868 (O-Me), 1598 (Ar), 1327 ( $\left.\mathrm{SO}_{2}\right), 1155$ ( $\left.\mathrm{SO}_{2}\right), 1092$ (SO), 813 (p-substitution) $\mathrm{cm}^{-1}$; major trans $\delta_{\mathrm{H}}\left(400 \mathrm{MHz}, \mathrm{CDCl}_{3}\right) 7.72(2 \mathrm{H}, \mathrm{d}, J 8.6 \mathrm{~Hz}, \mathrm{ArH}), 7.31(2 \mathrm{H}, \mathrm{d}, J$ $8.4 \mathrm{~Hz}, \mathrm{ArH}), 7.29$ (2H, d, J 8.4 Hz, ArH), 6.85 (2H, d, J $8.6 \mathrm{~Hz}, \mathrm{ArH}), 5.42$ (1H, dd, $J 5.5$ and $1.6 \mathrm{~Hz}, \mathrm{NH}), 3.80\left(3 \mathrm{H}, \mathrm{s}, \mathrm{OCH}_{3}\right), 3.31(1 \mathrm{H}, \mathrm{d}, J 3.9 \mathrm{~Hz}, \mathrm{NCHPh}), 3.10-3.01$ $(1 \mathrm{H}, \mathrm{m}, \mathrm{NCHH}), 2.99-2.93(1 \mathrm{H}, \mathrm{m}, \mathrm{NCHH}), 2.89-2.85(1 \mathrm{H}, \mathrm{m}, \mathrm{CHNCHPh}), 2.41$ $\left(3 \mathrm{H}, \mathrm{s}, \mathrm{CH}_{3}\right), 1.93-1.87\left(1 \mathrm{H}, \mathrm{m}, \mathrm{CHHCH}_{2}\right), 1.80-1.63(3 \mathrm{H}, \mathrm{m}, \mathrm{CH} H \mathrm{CH}), 1.12[9 \mathrm{H}$, s, $\left.\mathrm{C}\left(\mathrm{CH}_{3}\right)_{3}\right] ; \delta_{\mathrm{c}}\left(100.5 \mathrm{MHz}, \mathrm{CDCl}_{3}\right) 154.2(\mathrm{~s}), 143.4(\mathrm{~s}), 136.2(\mathrm{~s}), 132.0(\mathrm{~s}), 129.8$ (d), 129.7 (d), 127.6 (d), 127.1 (d), 83.9 (q), 57.9 (s), 56.2 (d), 47.9 (d), 42.5 (t), 34.4 (t), $24.6(\mathrm{t}), 22.3(\mathrm{q}), 21.5(\mathrm{q})$; $\underline{\text { minor trans }} \delta_{\mathrm{H}}\left(400 \mathrm{MHz}, \mathrm{CDCl}_{3}\right) 7.73(2 \mathrm{H}, \mathrm{d}, J 8.8$ $\mathrm{Hz}, \mathrm{ArH}), 7.62$ (2H, d, J 8.3 Hz, ArH), 7.30 (2H, d, J $8.3 \mathrm{~Hz}, \mathrm{ArH}), 7.15$ (2H, d, J 8.8 $\mathrm{Hz}, \mathrm{ArH}), 4.83\left(1 \mathrm{H}\right.$, br m, NH), $3.83\left(3 \mathrm{H}, \mathrm{s}, \mathrm{OCH}_{3}\right), 3.35$ (1H, d, J 4.0 Hz, NCHPh), 3.10-3.01 (1H, m, $\mathrm{NCHH}), 2.99-2.93(1 \mathrm{H}, \mathrm{m}, \mathrm{NCHH}), 2.41-2.35\left(4 \mathrm{H}, \mathrm{m}, \mathrm{CH}_{3}\right.$ and $\left.\mathrm{NCHCH}_{2}\right), 1.93-1.87\left(1 \mathrm{H}, \mathrm{m}, \mathrm{CHHCH} \mathrm{H}_{2}\right), 1.80-1.63(3 \mathrm{H}, \mathrm{m}, \mathrm{CH} H \mathrm{CHH}), 1.12$ [9H, s, $\left.\mathrm{C}\left(\mathrm{CH}_{3}\right)_{3}\right] ; \delta_{\mathrm{c}}\left(100.5 \mathrm{MHz}, \mathrm{CDCl}_{3}\right) 154.2$ (s), 143.4 (s), 136.2 (s), 132.0 (s), 129.8 (d), 129.7 (d), 127.6 (d), 127.1 (d), 83.9 (q), 57.9 (s), 53.3 (d), 47.4 (d), 42.5 (t), 34.4 (t), 24.6 (t), 22.3 (q), 21.5 (q); $\underline{\text { cis }} \delta_{\mathrm{H}}\left(400 \mathrm{MHz}, \mathrm{CDCl}_{3}\right) 7.84(2 \mathrm{H}, \mathrm{d}, J 8.3 \mathrm{~Hz}, \mathrm{ArH}), 7.69$ (2H, d, J 8.4 Hz, ArH), 7.17 (2H, d, J 8.4 Hz, ArH), 6.99 (2H, d, J 8.3 Hz, ArH), 4.80 $(1 \mathrm{H}, \mathrm{t}, J 6.2 \mathrm{~Hz}, \mathrm{NH}), 3.81\left(3 \mathrm{H}, \mathrm{s}, \mathrm{OCH}_{3}\right), 3.75(1 \mathrm{H}, \mathrm{d}, J 6.9 \mathrm{~Hz}, \mathrm{NCHPh}), 3.10-3.01$ $(1 \mathrm{H}, \mathrm{m}, \mathrm{NCHH}), 2.99-2.93(1 \mathrm{H}, \mathrm{m}, \mathrm{NCHH}), 2.71-2.63(1 \mathrm{H}, \mathrm{m}, \mathrm{CHNCHPh}), 2.41$ $\left(3 \mathrm{H}, \mathrm{s}, \mathrm{CH}_{3}\right), 1.93-1.87\left(1 \mathrm{H}, \mathrm{m}, \mathrm{CHHCH}_{2}\right), 1.80-1.63(3 \mathrm{H}, \mathrm{m}, \mathrm{CHHCHH}), 1.12[9 \mathrm{H}$, $\left.\mathrm{s}, \mathrm{C}\left(\mathrm{CH}_{3}\right)_{3}\right] ; \delta_{\mathrm{c}}\left(100.5 \mathrm{MHz}, \mathrm{CDCl}_{3}\right) 154.2(\mathrm{~s}), 143.4(\mathrm{~s}), 136.2(\mathrm{~s}), 132.0(\mathrm{~s}), 129.8$ (d), 129.7 (d), 127.6 (d), 127.1 (d), 83.8 (q), 54.2 (s), 44.2 (d), 43.4 (d), 42.5 (t), 28.1 (t), 24.6 (t), 22.3 (q), 21.5 (q); MS (ESI): $m / z(\%) 487\left(\mathrm{M}+\mathrm{Na}^{+}, 28 \%\right), 465\left(\mathrm{MH}^{+}\right.$, $49 \%$ ) and $391\left(\mathrm{M}^{+}-\mathrm{Bu}^{\mathrm{t}}-\mathrm{O}, 100 \%\right)$; HRMS (ESI) found 487.1695. $\mathrm{C}_{23} \mathrm{H}_{32} \mathrm{~N}_{2} \mathrm{O}_{4} \mathrm{~S}_{2} \mathrm{Na}$ requires 487.1696 . 


\section{Reaction of aminal 4 with achiral sulfonium salt 10}

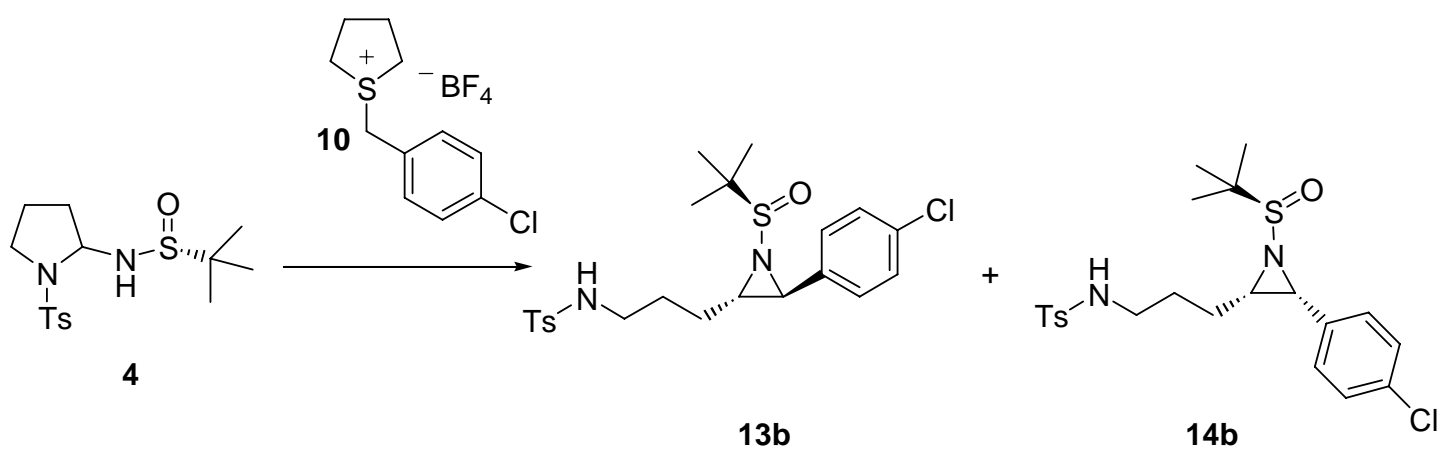

To a solution of N2-1-[(4-methylphenyl)sulfonyl]tetrahydro-1H-2-pyrrolyl-2methyl-2-propanesulfinamide $4(0.10 \mathrm{~g}, 0.29 \mathrm{mmol})$ in THF $(4 \mathrm{~mL})$ at $0{ }^{\circ} \mathrm{C}$ was added $\mathrm{P}_{2}$ base $(0.10 \mathrm{~mL}, 0.3 \mathrm{mmol})$ and the reaction mixture was left stirring for $30 \mathrm{~min} .1$ 4-Chlorobenzyltetrahydrothiophenium tetrafluoroborate $11(0.13 \mathrm{~g}, 0.44 \mathrm{mmol})$ was added at $0{ }^{\circ} \mathrm{C}$ followed by $\mathrm{P}_{2}$ base $(0.10 \mathrm{~mL}, 0.3 \mathrm{mmol})$. The reaction mixture was stirred for 3.5 hours at $0{ }^{\circ} \mathrm{C}$, diluted with $\mathrm{CH}_{2} \mathrm{Cl}_{2}(20 \mathrm{~mL})$ and $\mathrm{H}_{2} \mathrm{O}(3 \times 20 \mathrm{~mL})$. The aqueous layer was extracted with $\mathrm{CH}_{2} \mathrm{Cl}_{2}(3 \times 20 \mathrm{~mL})$. The combined organic layers were washed with brine $(20 \mathrm{~mL})$, and dried $\left(\mathrm{MgSO}_{4}\right)$. The solvents were removed in vacuo and the resultant residue was purified by column chromatography, eluting with 1:1 pet. ether:EtOAc to give:

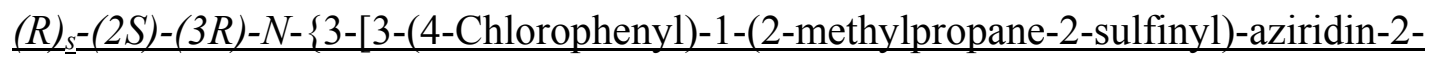
yl]-propyl -benzenesulfonamide (13b) as a white solid (60 mg, 50\%) mp 59-61 ${ }^{\circ} \mathrm{C}$ (pet. ether); $R_{\mathrm{f}}$ (EtOAc:pet. ether, 1:1) 0.35; IR (film), 1598 (Ar), $1327\left(\mathrm{SO}_{2}\right), 1158$ ( $\left.\mathrm{SO}_{2}\right), 1089$ (SO), 815 (p-substitution), $663(\mathrm{C}-\mathrm{Cl}) \mathrm{cm}^{-1}$; $\delta_{\mathrm{H}}\left(400 \mathrm{MHz}, \mathrm{CDCl}_{3}\right) 7.72$ $(2 \mathrm{H}, \mathrm{d}, J 8.3 \mathrm{~Hz}, \mathrm{ArH}), 7.33-7.26(4 \mathrm{H}, \mathrm{m}, \mathrm{ArH}), 7.12(2 \mathrm{H}, \mathrm{d}, J 8.3 \mathrm{~Hz}, \mathrm{ArH}), 5.28$ $(1 \mathrm{H}, \mathrm{t}, J 6.2 \mathrm{~Hz}, \mathrm{NH}), 3.11$ (1H, d, J 3.9 Hz, NCHPh), 3.01-2.89 (2H, m, $\left.\mathrm{NCH}_{2}\right), 2.47$ $(1 \mathrm{H}, \mathrm{td}, J 6.4$ and $3.9 \mathrm{~Hz}, \mathrm{CHNCHPh}), 2.41\left(3 \mathrm{H}, \mathrm{s}, \mathrm{CH}_{3}\right), 2.21-2.13(1 \mathrm{H}, \mathrm{m}$, $\left.\mathrm{CHHCH}_{2}\right), 2.04-1.95(1 \mathrm{H}, \mathrm{m}, \mathrm{CHHCHH}), 1.83-1.63(2 \mathrm{H}, \mathrm{m}, \mathrm{CH} H \mathrm{H} H \mathrm{H}), 1.19$ [9H, s, $\left.\mathrm{C}\left(\mathrm{CH}_{3}\right)_{3}\right] ; \delta_{\mathrm{c}}\left(100.5 \mathrm{MHz}, \mathrm{CDCl}_{3}\right) 143.3$ (s), 137.0 (s), 135.4 (s), 133.9 (s), 129.8 (d), 128.9 (d), 127.6 (d), 127.2 (d), 57.2 (s), 50.3 (d), 45.5 (d), $42.4(\mathrm{t}), 28.0$ (t), $25.6(\mathrm{t})$, 22.3 (q), 21.6 (q); MS (ESI): $m / z(\%) 491\left(\mathrm{M}+\mathrm{Na}^{+}, 17 \%\right)$ and $469\left(\mathrm{MH}^{+}, 100 \%\right)$; HRMS (ESI) found $469.1384 \mathrm{C}_{22} \mathrm{H}_{29} \mathrm{~N}_{2} \mathrm{O}_{3} \mathrm{~S}_{2} \mathrm{Cl}$ requires 469.1380 (Found: $\mathrm{C}, 56.68 \%$; $\mathrm{H}, 6.00 \%$; N, 5.74\%. $\mathrm{C}_{22} \mathrm{H}_{29} \mathrm{~N}_{2} \mathrm{O}_{3} \mathrm{~S}_{2} \mathrm{Cl}$ requires $\mathrm{C}, 56.33 \% ; \mathrm{H}, 6.23 \%$; N, 5.97\%); $[\alpha]_{\mathrm{D}}^{23}-133\left(c 0.75, \mathrm{CH}_{2} \mathrm{Cl}_{2}\right)$. 
$\underline{N-\{3-[3-(4-C h l o r o p h e n y l)-1-(2-m e t h y l p r o p a n e-2-s u l f i n y l)-a z i r i d i n-2-y l]-p r o p y l\}-~}$ benzenesulfonamide (13b minor:14b) (trans:cis) as a colourless oil (2:1) (19 mg, 16\%); $R_{\mathrm{f}}$ (EtOAc:pet. ether, 1:1) 0.50; IR (film), 1599 (Ar), $1329\left(\mathrm{SO}_{2}\right), 1161\left(\mathrm{SO}_{2}\right)$, 1091 (SO), 815 (p-substitution), $661(\mathrm{C}-\mathrm{Cl}) \mathrm{cm}^{-1} ; \underline{\text { trans }} \delta_{\mathrm{H}}\left(400 \mathrm{MHz}, \mathrm{CDCl}_{3}\right) 7.72$ (2H, d, J 8.3 Hz, ArH), 7.33-7.26 (4H, m, ArH), $7.12(2 \mathrm{H}, \mathrm{d}, J 8.3 \mathrm{~Hz}, \mathrm{ArH}), 4.86$ $(1 \mathrm{H}, \mathrm{t}, J 6.2 \mathrm{~Hz}, \mathrm{NH}), 3.34(1 \mathrm{H}, \mathrm{d}, J 3.7 \mathrm{~Hz}, \mathrm{NCHPh}), 2.99-2.93\left(2 \mathrm{H}, \mathrm{m}, \mathrm{NCH}_{2}\right)$, 2.41-2.35 (4H, m, $\mathrm{CH}_{3}$ and $\left.\mathrm{NCHCH}_{2}\right), 1.94-1.88\left(1 \mathrm{H}, \mathrm{m}, \mathrm{CHHCH}_{2}\right), 1.71-1.68(3 \mathrm{H}$, m, $\mathrm{CHHCHH}), 1.19$ [9H, s, C(CH3) $)_{3}$; $\delta_{\mathrm{c}}\left(100.5 \mathrm{MHz}, \mathrm{CDCl}_{3}\right) 143.5$ (s), 136.9 (s), 135.4 (s), 132.5 (s), 129.8 (d), 128.9 (d), 127.6 (d), 127.2 (d), 57.7 (s), 48.2 (d), 42.7 (d), $42.6(\mathrm{t}), 28.1(\mathrm{t}), 25.2(\mathrm{t}), 22.3(\mathrm{q}), 21.6(\mathrm{q}) ; \underline{c i s} \delta_{\mathrm{H}}\left(400 \mathrm{MHz}, \mathrm{CDCl}_{3}\right) 7.69(2 \mathrm{H}$, d, J 8.3 Hz, ArH), 7.33-7.26 (4H, m, ArH), $7.19(2 \mathrm{H}, \mathrm{d}, J 8.3 \mathrm{~Hz}, \mathrm{ArH}), 4.52(1 \mathrm{H}, \mathrm{t}, J$ $6.3 \mathrm{~Hz}, \mathrm{NH}), 3.75\left(1 \mathrm{H}, \mathrm{d}, J\right.$ 7.0 Hz, NCHPh), 2.87-2.79 (2H, m, $\left.\mathrm{NCH}_{2}\right), 2.41-2.35$ $\left(4 \mathrm{H}, \mathrm{m}, \mathrm{CH}_{3}\right.$ and $\left.\mathrm{NCHCH}_{2}\right), 1.94-1.88\left(1 \mathrm{H}, \mathrm{m}, \mathrm{CHHCH}_{2}\right), 1.71-1.68(3 \mathrm{H}, \mathrm{m}$, $\mathrm{CHHCHH}), 1.19$ [9H, s, C(CH$\left.)_{3}\right] ; \delta_{\mathrm{c}}\left(100.5 \mathrm{MHz} \mathrm{CDCl}_{3}\right) 143.5$ (s), 136.9 (s), 135.4 (s), 132.5 (s), 129.8 (d), 128.9 (d), 127.6 (d), 127.2 (d), 58.1 (s), 48.0 (d), 42.6 (t), 39.9 (d), 28.0 (t), 25.2 (t), 22.3 (q), 21.6 (q); MS (CI): $m / z(\%) 469\left(\mathrm{MH}^{+}, 62 \%\right)$ and $224\left(\mathrm{M}^{+}-\mathrm{NHSOBu}-\mathrm{CHPhCl}, 100 \%\right)$; HRMS (CI) found $469.1385 \mathrm{C}_{22} \mathrm{H}_{29} \mathrm{~N}_{2} \mathrm{O}_{3} \mathrm{~S}_{2} \mathrm{Cl}$ requires 469.1380 . 


\section{Reaction of aminal 4 with achiral sulfonium salt 11}
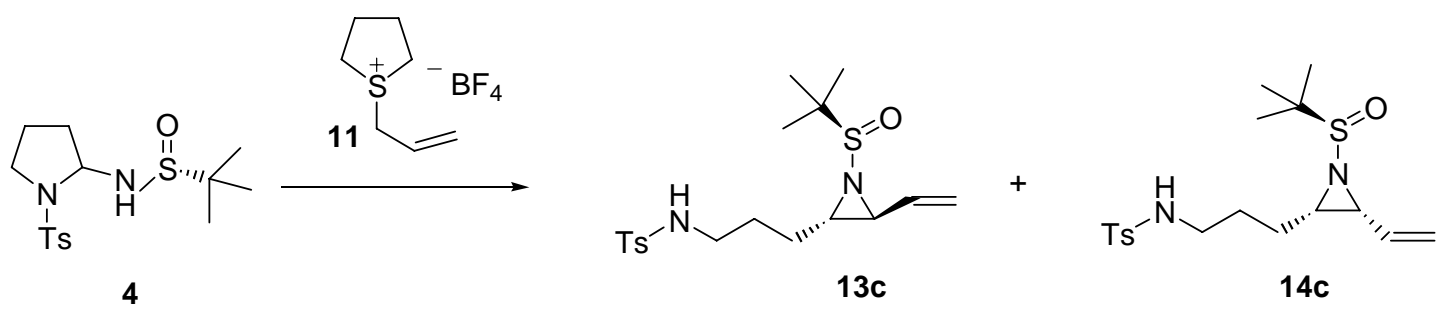

To a solution of N2-1-[(4-methylphenyl)sulfonyl]tetrahydro-1H-2-pyrrolyl-2methyl-2-propanesulfinamide $4(0.10 \mathrm{~g}, 0.29 \mathrm{mmol})$ in THF $(4 \mathrm{~mL})$ at $0{ }^{\circ} \mathrm{C}$ was added $\mathrm{P}_{2}$ base $(0.10 \mathrm{~mL}, 0.3 \mathrm{mmol})$ and the reaction mixture was left stirring for $30 \mathrm{~min}$. 1Allyltetrahydrothiophenium tetrafluoroborate $12(0.09 \mathrm{~g}, 0.44 \mathrm{mmol})$ was added at 0 ${ }^{\circ} \mathrm{C}$ followed by $\mathrm{P}_{2}$ base $(0.10 \mathrm{~mL}, 0.3 \mathrm{mmol})$. The reaction mixture was then stirred for 3.5 hours at $0{ }^{\circ} \mathrm{C}$, diluted with $\mathrm{CH}_{2} \mathrm{Cl}_{2}(20 \mathrm{~mL})$ and $\mathrm{H}_{2} \mathrm{O}(20 \mathrm{~mL})$. The aqueous layer was extracted with $\mathrm{CH}_{2} \mathrm{Cl}_{2}(3 \times 20 \mathrm{~mL})$. The combined organic layers were dried $\left(\mathrm{MgSO}_{4}\right)$. The solvents were removed in vacuo and the resultant residue was purified by column chromatography, eluting with 1:1 pet. ether:EtOAc to give:

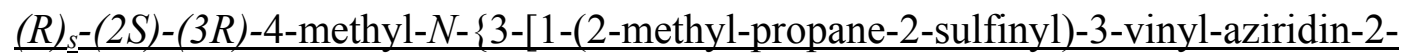
yll-propyl -benzenesulfonamide (13c) as a colourless oil (45 $\mathrm{mg}, 41 \%) ; R_{\mathrm{f}}$ (EtOAc:pet. ether, 1:1) 0.45; IR (film) 3054 (NH), $1161\left(\mathrm{SO}_{2}\right), 1093$ (SO), 903 $\left(\mathrm{C}=\mathrm{CH}_{2}\right), 896\left(\mathrm{C}=\mathrm{CH}_{2}\right) \mathrm{cm}^{-1} ; \delta_{\mathrm{H}}\left(400 \mathrm{MHz}, \mathrm{CDCl}_{3}\right) 7.79(2 \mathrm{H}, \mathrm{d}, J 8.3 \mathrm{~Hz}, \mathrm{ArH}), 7.32$ $(2 \mathrm{H}, \mathrm{d}, J 8.3 \mathrm{~Hz}, \mathrm{ArH}), 5.79\left(1 \mathrm{H}, \mathrm{ddd}, J 17.1,10.2\right.$ and $\left.7.9 \mathrm{~Hz}, \mathrm{CH}=\mathrm{CH}_{2}\right), 5.38(1 \mathrm{H}$, d, $J 17.1 \mathrm{~Hz}, \mathrm{CH}=\mathrm{CHH}), 5.20(1 \mathrm{H}, \mathrm{d}, J 10.2 \mathrm{~Hz}, \mathrm{CH}=\mathrm{C} H \mathrm{H}), 4.81(1 \mathrm{H}, \mathrm{t}, J 6.2 \mathrm{~Hz}$, $\mathrm{NH}), 3.05-2.97\left(2 \mathrm{H}, \mathrm{m}, \mathrm{NCH}_{2}\right), 2.78(1 \mathrm{H}, \mathrm{dd}, J 7.9$ and $3.9 \mathrm{~Hz}, \mathrm{NCHC}=\mathrm{C}), 2.41-2.35$ $\left(4 \mathrm{H}, \mathrm{m}, \mathrm{CH}_{3}\right.$ and $\left.\mathrm{NCHCH}_{2}\right), 1.77-1.58\left(4 \mathrm{H}, \mathrm{m}, \mathrm{CH}_{2} \mathrm{CH}_{2}\right), 1.23\left[9 \mathrm{H}, \mathrm{s}, \mathrm{C}_{\left.\left(\mathrm{CH}_{3}\right)_{3}\right] ; \delta_{\mathrm{c}}}\right.$ (100.5 MHz, $\left.\mathrm{CDCl}_{3}\right) 149.7$ (d), 143.5 (s), 135.8 (s), 129.8 (d), 127.2 (d), 120.2 (t), 57.4 (s), 50.2 (d), 42.7 (t), 41.9 (d), 28.8 (t), 26.2 (t), 22.6 (q), 21.6 (q); MS (CI): $\mathrm{m} / \mathrm{z}$ (\%) $385\left(\mathrm{MH}^{+}, 59 \%\right)$ and $224\left(\mathrm{M}^{+}-\mathrm{HNSOBu}-\mathrm{CHCH}=\mathrm{CH}_{2}, 100 \%\right)$; HRMS (CI) found 385.1619. $\mathrm{C}_{18} \mathrm{H}_{28} \mathrm{~N}_{2} \mathrm{O}_{3} \mathrm{~S}_{2}$ requires 385.1613; $[\alpha]_{\mathrm{D}}{ }^{23}-160\left(c 0.1, \mathrm{CH}_{2} \mathrm{Cl}_{2}\right)$.

$(R)_{s}-(2 S)-(3 S)-4-m e t h y l-N$ - $\{3-[1-(2-m e t h y l-p r o p a n e-2-s u l f i n y l)-3-v i n y l-a z i r i d i n-2-y l]-$ propyl \}-benzenesulfonamide (14c) as a colourless oil ( always obtained as a mixture with trans) (35 mg, 32\%); $R_{\mathrm{f}}$ (EtOAc:pet. ether, 1:1) 0.50; IR (film) $1163\left(\mathrm{SO}_{2}\right), 903$ 
$\left(\mathrm{C}=\mathrm{CH}_{2}\right), 896\left(\mathrm{C}=\mathrm{CH}_{2}\right) \mathrm{cm}^{-1} ; \delta_{\mathrm{H}}\left(400 \mathrm{MHz}, \mathrm{CDCl}_{3}\right) 7.73(2 \mathrm{H}, \mathrm{d}, J 8.3 \mathrm{~Hz}, \mathrm{ArH})$, $7.27(2 \mathrm{H}, \mathrm{d}, J 8.3 \mathrm{~Hz}, \mathrm{ArH}), 5.79\left(1 \mathrm{H}, \mathrm{ddd}, J 17.2,10.3\right.$ and $\left.7.4 \mathrm{~Hz}, \mathrm{CH}=\mathrm{CH}_{2}\right), 5.37$ $(1 \mathrm{H}, \mathrm{d}, J 17.2 \mathrm{~Hz}, \mathrm{CH}=\mathrm{CHH}), 5.31(1 \mathrm{H}, \mathrm{d}, J 10.3 \mathrm{~Hz}, \mathrm{CH}=\mathrm{CHH}), 4.68(1 \mathrm{H}, \mathrm{t}, J 6.3$ $\mathrm{Hz}, \mathrm{NH}), 3.16(1 \mathrm{H}, \mathrm{t}, \mathrm{J} 7.4 \mathrm{~Hz}, \mathrm{NCHC}=\mathrm{C}), 3.01-2.92\left(2 \mathrm{H}, \mathrm{m}, \mathrm{NCH}_{2}\right), 2.41(3 \mathrm{H}, \mathrm{s}$, $\left.\mathrm{CH}_{3}\right), 2.20\left(1 \mathrm{H}, \mathrm{td}, J 7.4\right.$ and $\left.5.5 \mathrm{~Hz}, \mathrm{NCHCH}_{2}\right), 1.62-1.42\left(4 \mathrm{H}, \mathrm{m}, \mathrm{CH}_{2} \mathrm{CH}_{2}\right), 1.17$ [9H, s, C(CH $\left.)_{3}\right] ; \delta_{\mathrm{c}}\left(100.5 \mathrm{MHz}, \mathrm{CDCl}_{3}\right) 143.4$ (d), 135.9 (s), 131.5 (s), 129.8 (d), $127.1(\mathrm{~d}), 120.9(\mathrm{t}), 56.8(\mathrm{~s}), 42.6(\mathrm{t}), 38.6(\mathrm{~d}), 35.9(\mathrm{~d}), 27.2(\mathrm{t}), 24.3(\mathrm{t}), 22.7(\mathrm{q})$, 21.5 (q); MS (ESI): $m / z(\%) 407\left(\mathrm{M}+\mathrm{Na}^{+}, 42 \%\right)$ and $385\left(\mathrm{MH}^{+}, 100 \%\right)$; HRMS (ESI) found 407.1435. $\mathrm{C}_{18} \mathrm{H}_{28} \mathrm{~N}_{2} \mathrm{O}_{3} \mathrm{~S}_{2} \mathrm{Na}$ requires 407.1434.

\section{Reaction of sulfinylimine 4 with achiral sulfonium salt 12}

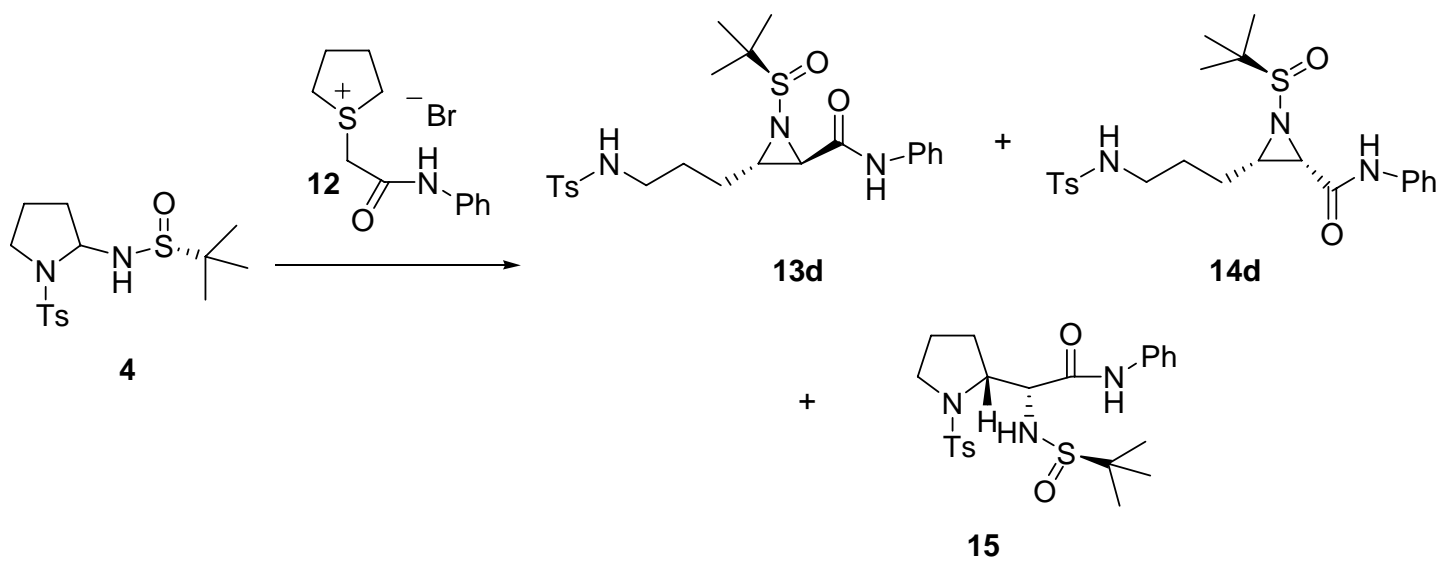

To a solution of N2-1-[(4-methylphenyl)sulfonyl]tetrahydro-1H-2-pyrrolyl-2methyl-2-propanesulfinamide $4(0.10 \mathrm{~g}, 0.29 \mathrm{mmol})$ in THF $(4 \mathrm{~mL})$ at $0{ }^{\circ} \mathrm{C}$ was added $\mathrm{P}_{2}$ base $(0.10 \mathrm{~mL}, 0.3 \mathrm{mmol})$ and the reaction mixture was left stirring for $30 \mathrm{~min} .1$ Phenylcarbamoylmethyltetrahydrothiophenium bromide $12(0.13 \mathrm{~g}, 0.44 \mathrm{mmol})$ was added at $0{ }^{\circ} \mathrm{C}$ followed by $\mathrm{P}_{2}$ base $(0.10 \mathrm{~mL}, 0.3 \mathrm{mmol})$. The reaction mixture was stirred for 3.5 hours at $0{ }^{\circ} \mathrm{C}$. If the reaction is stopped and quenched, a mixture of all three compounds is obtained (aziridines 63\% 1:4 trans:cis and 29\% pyrrolidine 15). The reaction was diluted with $\mathrm{CH}_{2} \mathrm{Cl}_{2}(20 \mathrm{~mL})$ and $\mathrm{H}_{2} \mathrm{O}(20 \mathrm{~mL})$. The aqueous layer was extracted with $\mathrm{CH}_{2} \mathrm{Cl}_{2}(3 \times 20 \mathrm{~mL})$. The combined organic layers were washed with brine $(20 \mathrm{~mL})$, and dried $\left(\mathrm{MgSO}_{4}\right)$. The solvents were removed in vacuo and the resultant residue was purified by column chromatography, eluting with 1:1 pet. ether:EtOAc. 
1-(2-Methyl-propane-2-sulfinyl)-3-[3-(toluene-4-sulfonylamino)propyl]-aziridine-2carboxylic acid phenylamide (13d:14d) (trans:cis) (1:4) as a colourless oil (87 mg, 63\%); $R_{\mathrm{f}}$ (EtOAc:pet. ether, 1:1) 0.30; IR (film) 3055 (NH), 1693 (NHCO), 1600 (Ar), 1526 (NHCO), $1327\left(\mathrm{SO}_{2}\right), 1160\left(\mathrm{SO}_{2}\right), 1027(\mathrm{SO}) \mathrm{cm}^{-1}$; trans $\delta_{\mathrm{H}}\left(400 \mathrm{MHz}, \mathrm{CDCl}_{3}\right)$ $8.66(1 \mathrm{H}, \mathrm{s}, \mathrm{NHCO}), 7.73(2 \mathrm{H}, \mathrm{d}, J 8.3 \mathrm{~Hz}, \mathrm{ArH}), 7.50(2 \mathrm{H}, \mathrm{dd}, J 7.8$ and $1.6 \mathrm{~Hz}$, ArH), 7.35-7.21 (4H, m, ArH), $7.07(1 \mathrm{H}, \mathrm{tt}, J 7.8$ and $1.6 \mathrm{~Hz}, \mathrm{ArH}), 5.25(1 \mathrm{H}, \mathrm{t}, J 6.3$ $\mathrm{Hz}, \mathrm{NH}), 3.23$ (1H, d, J $3.7 \mathrm{~Hz}, \mathrm{NCHCO}), 3.02-2.91\left(2 \mathrm{H}, \mathrm{m}, \mathrm{NCH}_{2}\right), 2.82(1 \mathrm{H}, \mathrm{ddd}, J$ 6.9, 5.5 and $3.7 \mathrm{~Hz}, \mathrm{CHNCHCO}), 2.39\left(3 \mathrm{H}, \mathrm{s}, \mathrm{CH}_{3}\right), 1.91-1.82\left(1 \mathrm{H}, \mathrm{m}, \mathrm{CH} \mathrm{HCH}_{2}\right)$, 1.78-1.69 (3H, m, CHHCHH), $1.30\left[9 \mathrm{H}, \mathrm{s}, \mathrm{C}\left(\mathrm{CH}_{3}\right)_{3}\right]$; $\delta_{\mathrm{c}}\left(100.5 \mathrm{MHz}, \mathrm{CDCl}_{3}\right) 165.1$ (s), 143.5 (s), 137.2 (s), 136.9 (s), 129.8 (d), 129.2 (d), 127.1 (d), 124.9 (d), 119.9 (d), $57.6(\mathrm{~s}), 46.1$ (d), 44.0 (d), $42.3(\mathrm{t}), 27.4(\mathrm{t}), 24.4(\mathrm{t}), 22.5$ (q), $21.6(\mathrm{q}) ; \underline{c i s} \delta_{\mathrm{H}}(400$ MHz, $\left.\mathrm{CDCl}_{3}\right) 8.36(1 \mathrm{H}, \mathrm{s}, \mathrm{NHCO}), 7.68(2 \mathrm{H}, \mathrm{d}, J 8.3 \mathrm{~Hz}, \mathrm{ArH}), 7.56(2 \mathrm{H}, \mathrm{dd}, J 8.6$ and 1.1 Hz, ArH), $7.33(2 \mathrm{H}, \mathrm{t}, J 8.6 \mathrm{~Hz}, \mathrm{ArH}), 7.24(2 \mathrm{H}, \mathrm{d}, J 8.3 \mathrm{~Hz}, \mathrm{ArH}), 7.14(1 \mathrm{H}$, tt, $J 8.6$ and $1.1 \mathrm{~Hz}, \mathrm{ArH}), 5.08(1 \mathrm{H}, \mathrm{t}, J 6.3 \mathrm{~Hz}, \mathrm{NH}), 3.42(1 \mathrm{H}, \mathrm{d}, J 7.3 \mathrm{~Hz}$, $\mathrm{NCHCO}), 3.08-2.95\left(2 \mathrm{H}, \mathrm{m}, \mathrm{NCH}_{2}\right), 2.43(1 \mathrm{H}, \mathrm{ddd}, J 7.3,6.8$ and $4.9 \mathrm{~Hz}$, $\mathrm{CHNCHCO}), 2.39\left(3 \mathrm{H}, \mathrm{s}, \mathrm{CH}_{3}\right), 1.75-1.63(4 \mathrm{H}, \mathrm{m}, \mathrm{CH} H \mathrm{CH}), 1.28[9 \mathrm{H}, \mathrm{s}$, $\left.\mathrm{C}\left(\mathrm{CH}_{3}\right)_{3}\right] ; \delta_{\mathrm{c}}\left(100.5 \mathrm{MHz}, \mathrm{CDCl}_{3}\right) 164.5$ (s), 143.5 (s), 137.0 (s), 136.9 (s), 129.8 (d), 129.2 (d), 127.1 (d), 125.0 (d), 119.9 (d), 57.6 (s), 42.3 (t), 39.5 (d), 35.8 (d), 27.2 (t), 23.9 (t), 22.6 (q), 21.6 (q); MS (CI): m/z (\%) $478\left(\mathrm{MH}^{+}, 20 \%\right), 253\left(\mathrm{MH}^{+}-\right.$ TsNHCH $\left.\mathrm{CH}_{2} \mathrm{CH}_{2} \mathrm{CH}, 48 \%\right), 224\left(\mathrm{M}^{+}-\mathrm{HNSOBu}-\mathrm{CHCONHPh}, 71 \%\right)$ and $57\left(\mathrm{Bu}^{\mathrm{t}+}\right.$, $100 \%$ ); cis isomer: HRMS (CI) found $478.1834 \mathrm{C}_{23} \mathrm{H}_{31} \mathrm{~N}_{3} \mathrm{O}_{4} \mathrm{~S}_{2}$ requires 478.1833; $[\alpha]_{\mathrm{D}}^{23}-110\left(c 0.1, \mathrm{CH}_{2} \mathrm{Cl}_{2}\right)$.

If the reaction is left stirring for 2 more days, then a mixture of cis aziridine 14d and pyrrolidine 15 is obtained (cis aziridine $45 \%$ and $47 \%$ pyrrolidine 15): $(R)_{\underline{s}}-(R)-\alpha-$ (2R)-2-(2-Methyl-propane-2-sulfinylamino)-N phenyl-2-[1-(toluene-4-sulfonyl)pyrrolodon-2-yl]-acetamide (15) as a yellow oil (65 mg, 47\%); $R_{\mathrm{f}}($ EtOAc:pet. ether, 1:1) 0.30; IR (film) 3055 (NH), 1693 (NHCO), 1600 (Ar), 1526 (NHCO), 1327 ( $\left.\mathrm{SO}_{2}\right)$, $1160\left(\mathrm{SO}_{2}\right), 1027(\mathrm{SO}) \mathrm{cm}^{-1} ; \delta_{\mathrm{H}}\left(400 \mathrm{MHz}, \mathrm{CDCl}_{3}\right) 8.53(1 \mathrm{H}, \mathrm{s}, \mathrm{NHCO}), 7.73(2 \mathrm{H}, \mathrm{d}$, $J 8.3 \mathrm{~Hz}, \mathrm{ArH}), 7.50$ (2H, dd, $J .8$ and 1.6 Hz, ArH), 7.35-7.21 (4H, m, ArH), 7.14 $(1 \mathrm{H}, \mathrm{tt}, J 7.8$ and $1.6 \mathrm{~Hz}, \mathrm{ArH}), 5.84(1 \mathrm{H}, \mathrm{d}, J 6.4 \mathrm{~Hz}, \mathrm{NH}), 4.20(1 \mathrm{H}, \mathrm{dd}, J 6.4$ and $2.1 \mathrm{~Hz}, \mathrm{NCHCO}), 3.41-3.27\left(2 \mathrm{H}, \mathrm{m}, \mathrm{NCH}_{2}\right), 2.45-2.41(4 \mathrm{H}, \mathrm{m}, \mathrm{NCHCHCO}$ and $\left.\mathrm{CH}_{3}\right), 1.91-1.69\left(4 \mathrm{H}, \mathrm{m}, \mathrm{CH}_{2} \mathrm{CH}_{2}\right), 1.39\left[9 \mathrm{H}, \mathrm{s}, \mathrm{C}\left(\mathrm{CH}_{3}\right)_{3}\right] ; \delta_{\mathrm{c}}\left(100.5 \mathrm{MHz}, \mathrm{CDCl}_{3}\right)$ 168.2 (s), 144.4 (s), 137.3 (s), 133.7 (s), 129.8 (d), 129.0 (d), 127.8 (d), 124.7 (d), 
119.7 (d), 64.7 (d), 62.9 (d), 56.7 (s), 51.3 (t), 30.1 (t), 26.0 (t), 22.9 (q), 21.7 (q); MS (CI): $m / z(\%) 478\left(\mathrm{MH}^{+}, 20 \%\right), 253\left(\mathrm{MH}^{+}-\mathrm{TsNHCH}_{2} \mathrm{CH}_{2} \mathrm{CH}_{2} \mathrm{CH}, 48 \%\right), 224\left(\mathrm{M}^{+}-\right.$ $\left.\mathrm{HNSOBu}{ }^{\mathrm{t}}-\mathrm{CHCONHPh}, 71 \%\right)$ and $57\left(\mathrm{Bu}^{\mathrm{t}+}, 100 \%\right)$. HRMS (CI) found 478.1838 $\mathrm{C}_{23} \mathrm{H}_{31} \mathrm{~N}_{3} \mathrm{O}_{4} \mathrm{~S}_{2}$ requires $478.1833 ;[\alpha]_{\mathrm{D}}^{23}-40\left(c 1.0, \mathrm{CH}_{2} \mathrm{Cl}_{2}\right)$.

\section{$(R)_{\mathrm{s}}$-(2S)-(3S)-2-Methylpropane-2-sulfinic acid [2-phenyl-1-(toluene-4-sulfonyl)- piperidin-3-yl]-amide (16)}

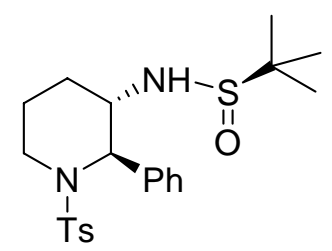

To a solution of 4-methyl- $N$-\{3-[3-phenyl-1-(propane-2-sulfinyl)-aziridin-2yl]propyl benzenesulfonamide $7(30 \mathrm{mg}, 0.07 \mathrm{mmol})$ in $\mathrm{CH}_{2} \mathrm{Cl}_{2}(2 \mathrm{~mL})$ at room temperature was added $\mathrm{Yb}(\mathrm{OTf})_{3}(50 \mathrm{mg}, 0.02 \mathrm{mmol})$. After $48 \mathrm{~h}$ the reaction was diluted with $\mathrm{CH}_{2} \mathrm{Cl}_{2}(10 \mathrm{~mL})$ and $\mathrm{H}_{2} \mathrm{O}(10 \mathrm{~mL})$. The aqueous layer was washed with $\mathrm{CH}_{2} \mathrm{Cl}_{2}(3 \times 10 \mathrm{~mL})$. The combined organic layers were washed with brine $(20 \mathrm{~mL})$, dried $\left(\mathrm{MgSO}_{4}\right)$ and the solvents were removed in vacuo. The crude product purified by column chromatography, eluting with 1:1 pet. ether:EtOAc to give the product as a white solid (25 mg, 93\%) mp 79-82 ${ }^{\circ} \mathrm{C}$ (pet. ether); $R_{\mathrm{f}}$ (EtOAc:pet. ether, 1:1) 0.25; IR (film) 1599 (Ar), $1326\left(\mathrm{SO}_{2}\right), 1159\left(\mathrm{SO}_{2}\right), 1058(\mathrm{SO}) \mathrm{cm}^{-1} ; \delta_{\mathrm{H}}\left(400 \mathrm{MHz}, \mathrm{CDCl}_{3}\right)$ $7.69(2 \mathrm{H}, \mathrm{d}, J 8.3 \mathrm{~Hz}, \mathrm{ArH}), 7.30-7.19(5 \mathrm{H}, \mathrm{m}, \mathrm{ArH}), 7.04(2 \mathrm{H}, \mathrm{d}, J 8.3 \mathrm{~Hz}, \mathrm{ArH})$, $5.21(1 \mathrm{H}, \mathrm{br} \mathrm{s}, \mathrm{NCHPh}), 4.17(1 \mathrm{H}, \mathrm{d}, J 8.2 \mathrm{~Hz}, \mathrm{NH}), 3.84(1 \mathrm{H}, \mathrm{ddd}, J 9.8,8.2$ and 2.0 $\mathrm{Hz}, \mathrm{NHCHCHPh}), 3.05$ (1H, td, $J 13.1$ and $2.5 \mathrm{~Hz}, \mathrm{NCHH}), 2.87(1 \mathrm{H}, \mathrm{dt}, J 13.1$ and $6.9 \mathrm{~Hz}, \mathrm{NCHH}), 2.41\left(3 \mathrm{H}, \mathrm{s}, \mathrm{CH}_{3}\right), 1.92-1.75(3 \mathrm{H}, \mathrm{m}, \mathrm{CHHCH}), 1.64-1.52(1 \mathrm{H}, \mathrm{m}$, $\mathrm{CHHCHH}), 1.24\left[9 \mathrm{H}, \mathrm{s}, \mathrm{C}\left(\mathrm{CH}_{3}\right)_{3}\right] ; \delta_{\mathrm{c}}\left(100.5 \mathrm{MHz}, \mathrm{CDCl}_{3}\right) 143.3$ (s), 138.2 (s), 136.5 (s), 129.8 (d), 128.8 (d), 127.4 (d), 127.0 (d), 126.8 (d), 61.2 (d), 59.1 (d), 56.0 (s), $50.6(\mathrm{t}), 24.3(\mathrm{t}), 23.8(\mathrm{t}), 22.8(\mathrm{q}), 21.5(\mathrm{q})$; MS (CI): $\mathrm{m} / z(\%) 435\left(\mathrm{MH}^{+}, 89 \%\right)$ and $224\left(\mathrm{M}^{+}-\mathrm{NSOBu}-\mathrm{CH}_{2} \mathrm{Ph}, 100 \%\right)$; HRMS (CI) found 435.1776. $\mathrm{C}_{22} \mathrm{H}_{30} \mathrm{~N}_{2} \mathrm{O}_{3} \mathrm{~S}_{2}$ requires 435.1769; $[\alpha]_{\mathrm{D}}^{23}-32\left(c 1.0, \mathrm{CH}_{2} \mathrm{Cl}_{2}\right)$. 


\section{(2R)-(3S)-trans 4-Methyl- $N$-[3-(3-phenylaziridin-2-yl)-propyl] \\ benzenesulfonamide (17)}

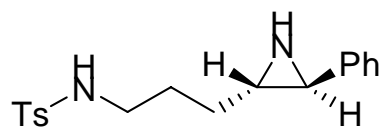

To a solution of 4-methyl- $N$-\{3-[3-phenyl-1-(propane-2-sulfinyl)-aziridin-2yl]-propyl $\}$-benzenesulfonamide 6 (20 $\mathrm{mg}, 0.04 \mathrm{mmol})$ in anhydrous 1,4-dioxane (1 $\mathrm{mL})$ at r.t. was added a solution of $\mathrm{HCl}(1.25 \mathrm{M})$ in EtOH. The reaction was monitored by TLC and the amount of $\mathrm{HCl}$ was determined by the reaction progress. Once all starting material was consumed, the reaction mixture was concentrated. The reaction mixture was diluted with $\mathrm{Et}_{2} \mathrm{O}(4 \mathrm{~mL})$ and washed with $\mathrm{H}_{2} \mathrm{O}(3 \times 4 \mathrm{~mL})$. The combined aqueous layers were basified with $\mathrm{NH}_{3}(1 \mathrm{~N}, 30 \mathrm{~mL})$, extracted with $\mathrm{Et}_{2} \mathrm{O}$ $(3 \times 25 \mathrm{~mL})$ and dried $\left(\mathrm{MgSO}_{4}\right)$. The solvents were removed in vacuo to afford the product as a white solid (11 mg, 81\%) mp 122-124 ${ }^{\circ} \mathrm{C}\left(\mathrm{CH}_{2} \mathrm{Cl}_{2}\right) ; R_{\mathrm{f}}\left(\mathrm{CH}_{2} \mathrm{Cl}_{2}: \mathrm{MeOH}\right.$, 9:1) 0.10; IR (film) 3379 (NH), $3324(\mathrm{NH}), 1597$ (Ar), $1330\left(\mathrm{SO}_{2}\right), 1163\left(\mathrm{SO}_{2}\right) \mathrm{cm}^{-1}$; $\delta_{\mathrm{H}}\left(400 \mathrm{MHz}, \mathrm{CDCl}_{3}\right) 7.68(2 \mathrm{H}, \mathrm{d}, J 8.3 \mathrm{~Hz}, \mathrm{ArH}), 7.28-7.19(5 \mathrm{H}, \mathrm{m}, \mathrm{ArH}), 7.17(2 \mathrm{H}$, d, $J 8.3 \mathrm{~Hz}, \mathrm{ArH}), 4.91(1 \mathrm{H}, \mathrm{d}, J 2.9 \mathrm{~Hz}, \mathrm{NCHPh}), 3.69(1 \mathrm{H}, \mathrm{dt}, J 13.3$ and $3.9 \mathrm{~Hz}$, $\mathrm{NCHH}), 3.54\left(1 \mathrm{H}, \mathrm{dd}, J 7.6\right.$ and $\left.2.9 \mathrm{~Hz}, \mathrm{NCHCH}_{2}\right), 3.19(1 \mathrm{H}$, ddd, $J 13.3,11.8$ and $3.4 \mathrm{~Hz}, \mathrm{NCHH}), 2.41\left(3 \mathrm{H}, \mathrm{s}, \mathrm{CH}_{3}\right), 1.85-1.74\left(1 \mathrm{H}, \mathrm{m}, \mathrm{CHHCH}_{2}\right), 1.68-1.57(3 \mathrm{H}, \mathrm{m}$, $\mathrm{CHHCHH}$ and $2 \mathrm{x} \mathrm{NH}), 1.53-1.47(1 \mathrm{H}, \mathrm{m}, \mathrm{CHHCHH}), 1.43-1.39(1 \mathrm{H}, \mathrm{m}$, $\mathrm{CHHCHH}) ; \delta_{\mathrm{c}}\left(100.5 \mathrm{MHz}, \mathrm{CDCl}_{3}\right) 143.3$ (s), 143.2 (s), 132.8 (s), 129.7 (d), 128.7 (d), 128.0 (d), 127.2 (d), 126.3 (d), 53.2 (d), 50.4 (d), 42.5 (t), 28.2 (t), 25.8 (t), 21.4 (q); $\operatorname{MS}(\mathrm{CI}): m / z(\%) 331\left(\mathrm{MH}^{+}, 98 \%\right), 314\left(\mathrm{MH}^{+}-\mathrm{NH}_{3}, 100 \%\right)$ and $175\left(\mathrm{M}^{+}-\mathrm{Ts}\right.$, 75\%); HRMS (CI) found 331.1480. $\mathrm{C}_{18} \mathrm{H}_{22} \mathrm{~N}_{2} \mathrm{O}_{2} \mathrm{~S}$ requires 331.1473; (Found: $\mathrm{C}$, $65.51 \% ; \mathrm{H}, 6.61 \% ; \mathrm{N}, 8.34 \% . \mathrm{C}_{18} \mathrm{H}_{22} \mathrm{~N}_{2} \mathrm{O}_{2} \mathrm{~S}$ requires $\mathrm{C}, 65.42 \% ; \mathrm{H}, 6.71 \% ; \mathrm{N}, 8.48 \%$ ) $[\alpha]_{\mathrm{D}}^{23}+40\left(c 0.1, \mathrm{CH}_{2} \mathrm{Cl}_{2}\right)$. 


\section{(2S)-(3S)-2-Phenyl-1-(toluene-4-sulfonyl)-piperidin-3-ylamine (18)}<smiles>N[C@@H]1CCCN([AsH2-])[C@@H]1c1ccccc1</smiles>

To a solution of 2-methylpropane-2-sulfinic acid [2-phenyl-1-(toluene-4sulfonyl)-piperidin-3-yl]-amide 16 (10 mg, $0.02 \mathrm{mmol})$ in anhydrous 1,4-dioxane (1 $\mathrm{mL})$ at room temperature was added a solution of $\mathrm{HCl}(1.25 \mathrm{M})$ in $\mathrm{EtOH}$. The reaction was monitored by $\mathrm{TLC}$ and the amount of $\mathrm{HCl}$ was determined by the reaction progress. Once all starting material was consumed, the reaction mixture was concentrated. The reaction mixture was diluted with $\mathrm{Et}_{2} \mathrm{O}(4 \mathrm{~mL})$ and washed with $\mathrm{H}_{2} \mathrm{O}(3 \times 4 \mathrm{~mL})$. The combined aqueous layers were basified with $\mathrm{NH}_{3}(1 \mathrm{~N}, 30 \mathrm{~mL})$, extracted with $\mathrm{Et}_{2} \mathrm{O}(3 \times 25 \mathrm{~mL})$ and dried $\left(\mathrm{MgSO}_{4}\right)$. The solvents were removed in vacuo to afford the product as a colourless oil (6 mg, $88 \%) ; R_{\mathrm{f}}\left(\mathrm{CH}_{2} \mathrm{Cl}_{2}: \mathrm{MeOH}, 9: 1\right)$ 0.15; IR (film) $3496(\mathrm{NH}), 1387\left(\mathrm{SO}_{2}\right), 1160\left(\mathrm{SO}_{2}\right) \mathrm{cm}^{-1} ; \delta_{\mathrm{H}}\left(400 \mathrm{MHz}, \mathrm{CDCl}_{3}\right) 7.68$ (2H, d, J 8.2 Hz, ArH), 7.43-7.19 (7H, m, ArH), 4.89 (1H, d, J 2.6 Hz, NCHPh), 3.84 $\left(1 \mathrm{H}\right.$, ddd, $J$ 7.7, 7.6 and $\left.2.6 \mathrm{~Hz}, \mathrm{NH}_{2} \mathrm{CH}\right), 3.26-3.18\left(2 \mathrm{H}, \mathrm{m}, \mathrm{NCH}_{2}\right), 2.41\left(3 \mathrm{H}, \mathrm{s}, \mathrm{CH}_{3}\right)$, 1.78-1.52 (6H, m, $\mathrm{CH}_{2} \mathrm{CH}_{2}$ and $\left.\mathrm{NH}_{2}\right) ; \delta_{\mathrm{c}}\left(100.5 \mathrm{MHz}, \mathrm{CDCl}_{3}\right) 145.0$ (s), 136.9 (s), 136.8 (s), 129.8 (d), 128.7 (d), 127.5 (d), 127.4 (d), 127.2 (d), 60.4 (d), 57.9 (d), 41.9 (t), $29.8(\mathrm{t}), 25.2(\mathrm{t}), 21.6(\mathrm{q})$; MS (ESI): $m / z(\%) 331\left(\mathrm{MH}^{+}, 100 \%\right)$; HRMS (ESI) found 331.1476 $\mathrm{C}_{18} \mathrm{H}_{22} \mathrm{~N}_{2} \mathrm{O}_{2} \mathrm{~S}$ requires 331.1474; (Found: $\mathrm{C}, 65.53 \%$; H, 6.62\%; N, $8.34 \% . \mathrm{C}_{18} \mathrm{H}_{22} \mathrm{~N}_{2} \mathrm{O}_{2} \mathrm{~S}$ requires $\left.\mathrm{C}, 65.42 \% ; \mathrm{H}, 6.71 \% ; \mathrm{N}, 8.48 \%\right) ;[\alpha]_{\mathrm{D}}^{23}+40(c 0.2$, $\mathrm{CH}_{2} \mathrm{Cl}_{2}$ ). 
${ }^{1} \mathrm{H}$ NMR $\left(\mathrm{CDCl}_{3}\right)$ Aminal 4

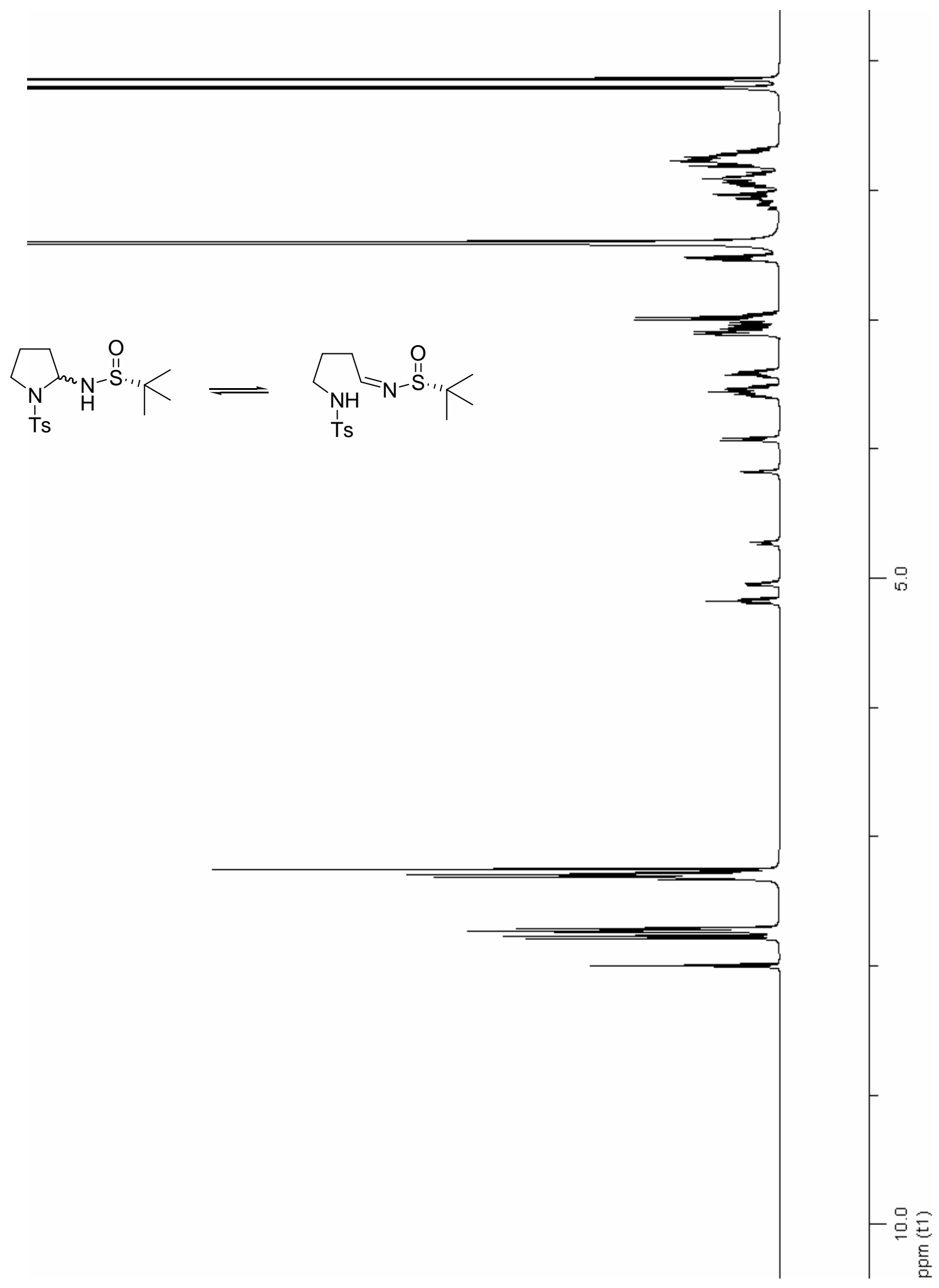


${ }^{13} \mathrm{C}$ NMR $\left(\mathrm{CDCl}_{3}\right)$ Aminal 4

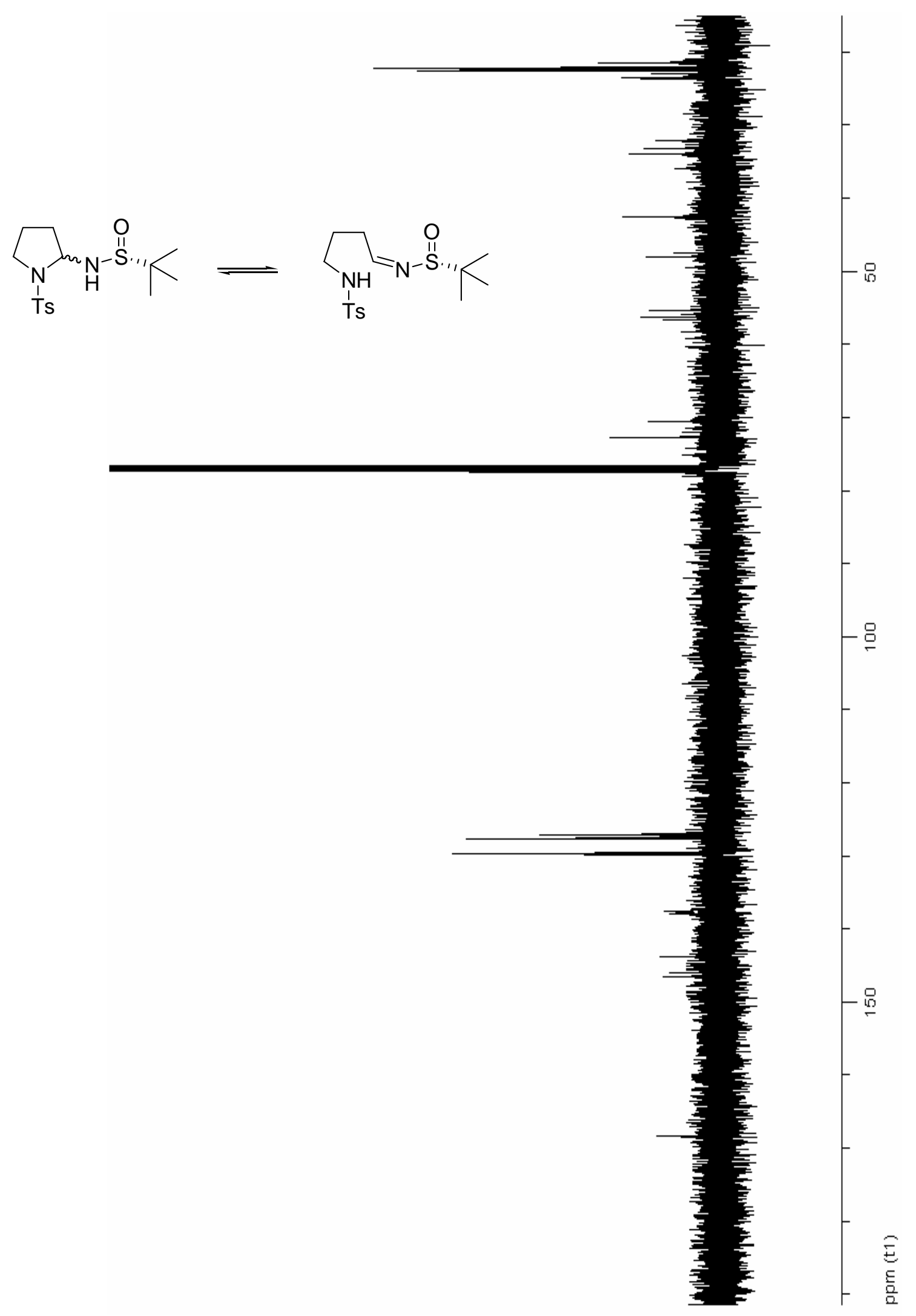


${ }^{1} \mathrm{H}$ NMR $\left(\mathrm{CDCl}_{3}\right)$ aziridines 6 minor: aziridine $\mathbf{7}$

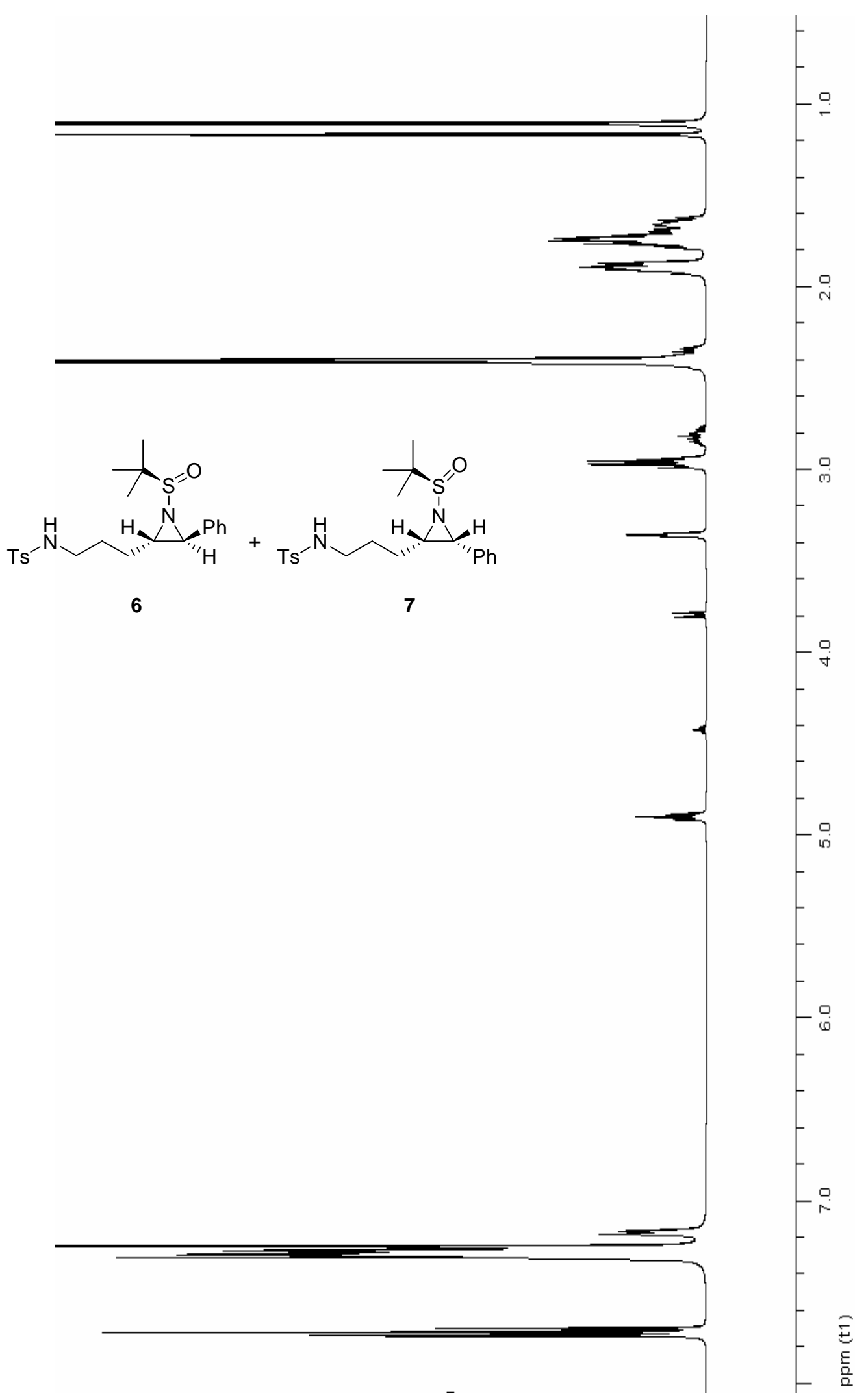


${ }^{13} \mathrm{C}$ NMR $\left(\mathrm{CDCl}_{3}\right)$ aziridines 6 minor: aziridine 7

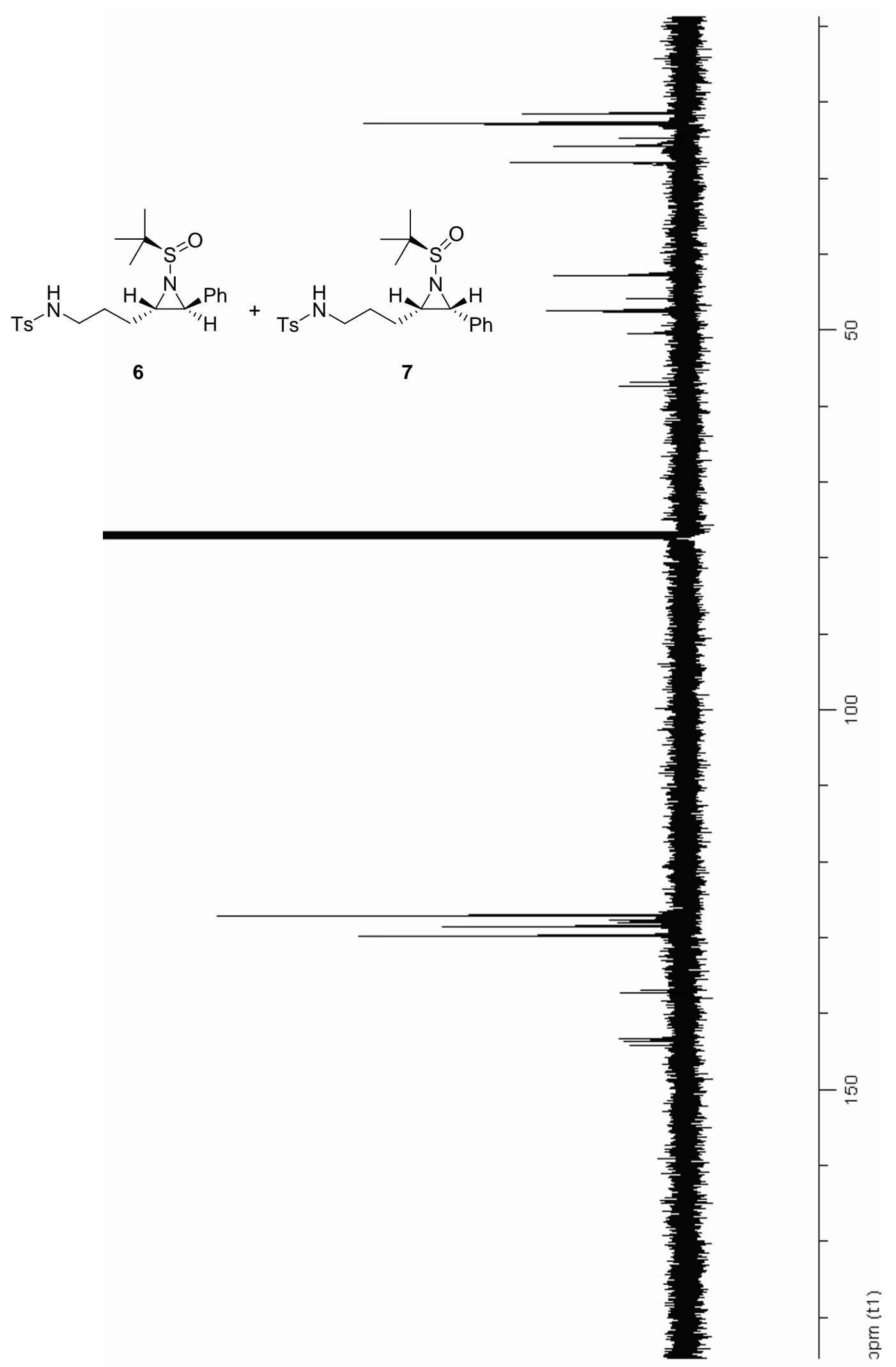


${ }^{1} \mathrm{H}$ NMR $\left(\mathrm{CDCl}_{3}\right)$ pyrrolidine 8

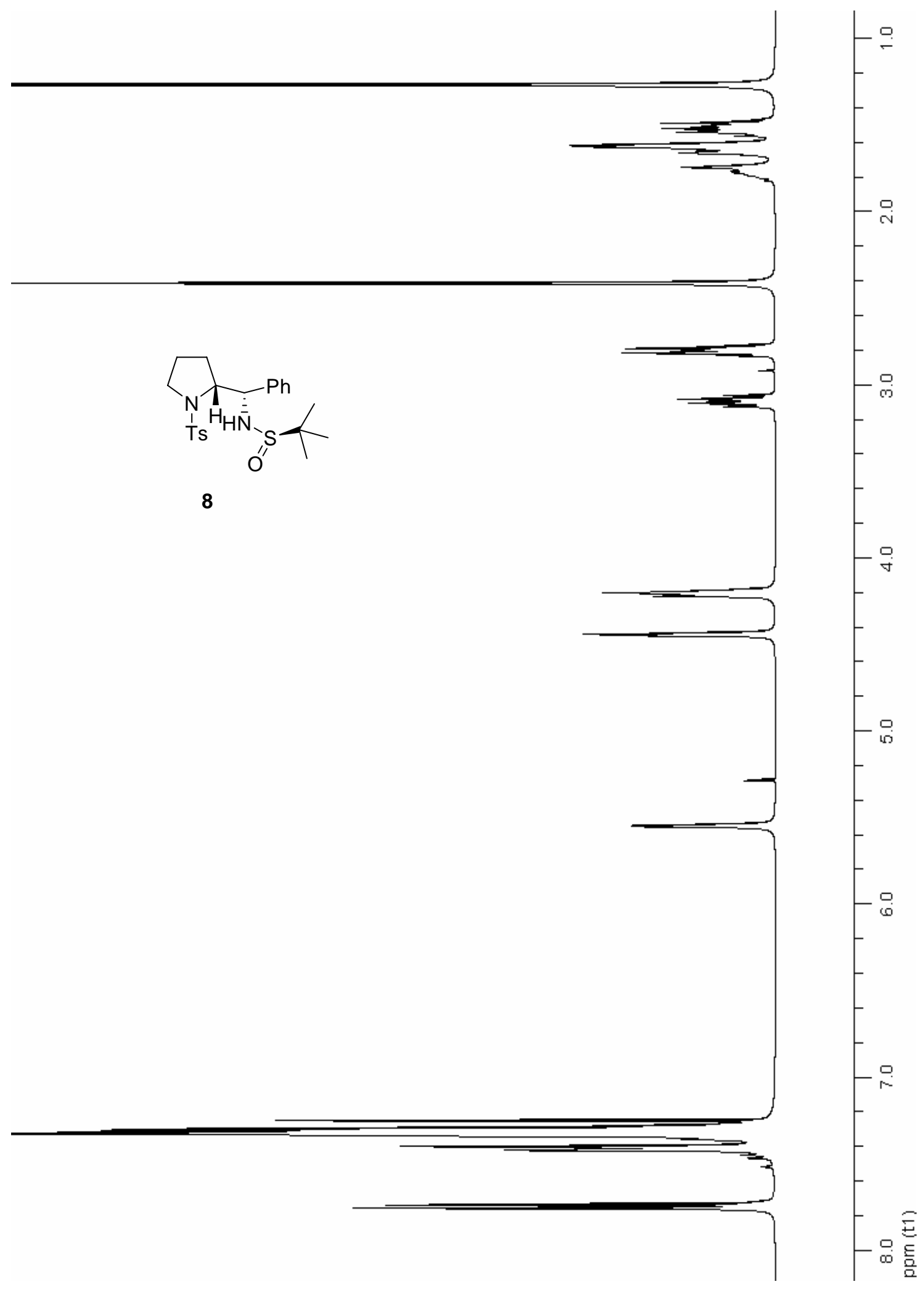


${ }^{13} \mathrm{C}$ NMR $\left(\mathrm{CDCl}_{3}\right)$ pyrrolidine 8

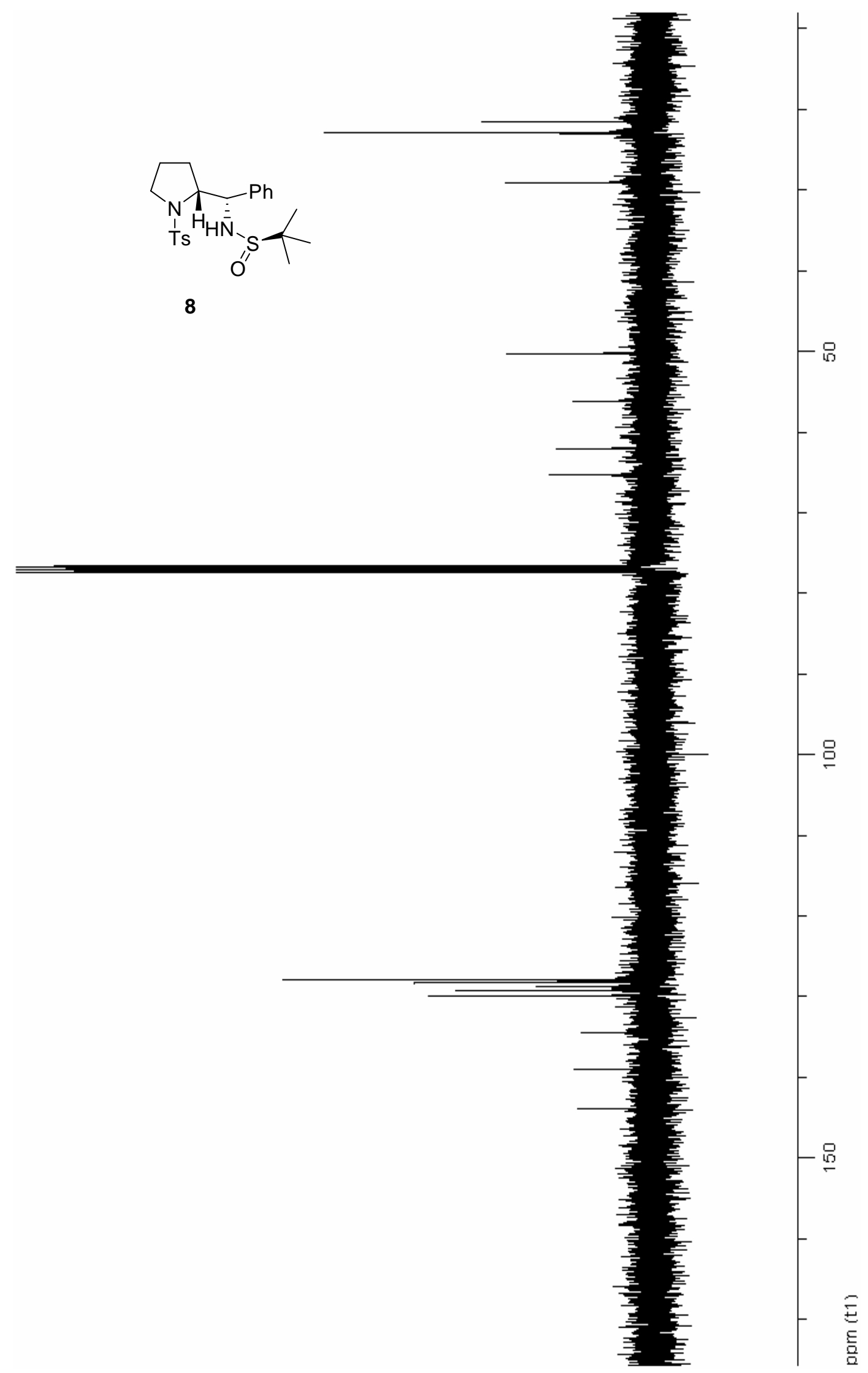


${ }^{1} \mathrm{H}$ NMR $\left(\mathrm{CDCl}_{3}\right)$ aziridines 6':7'

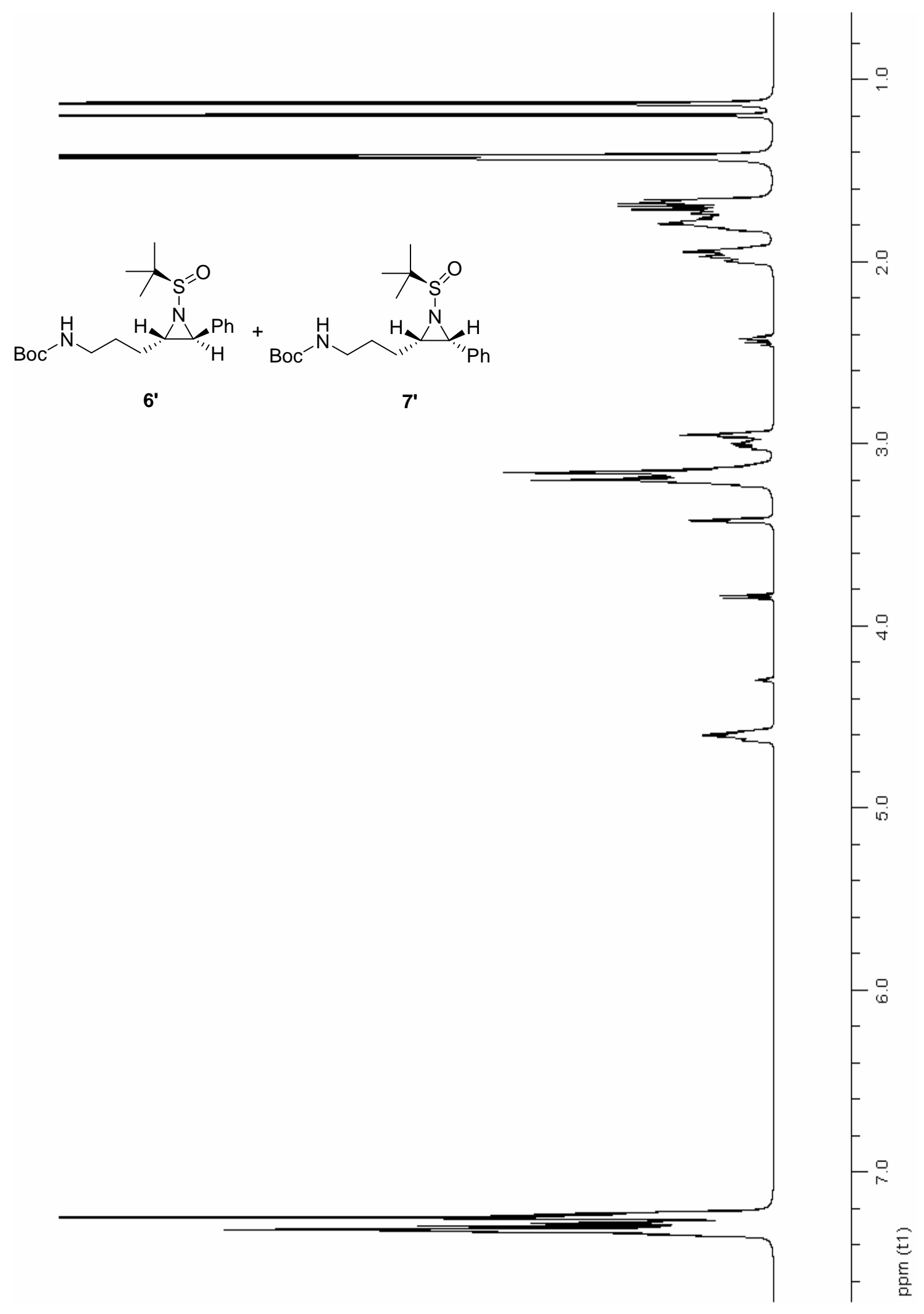


${ }^{13} \mathrm{C}$ NMR $\left(\mathrm{CDCl}_{3}\right)$ aziridines 6':7'

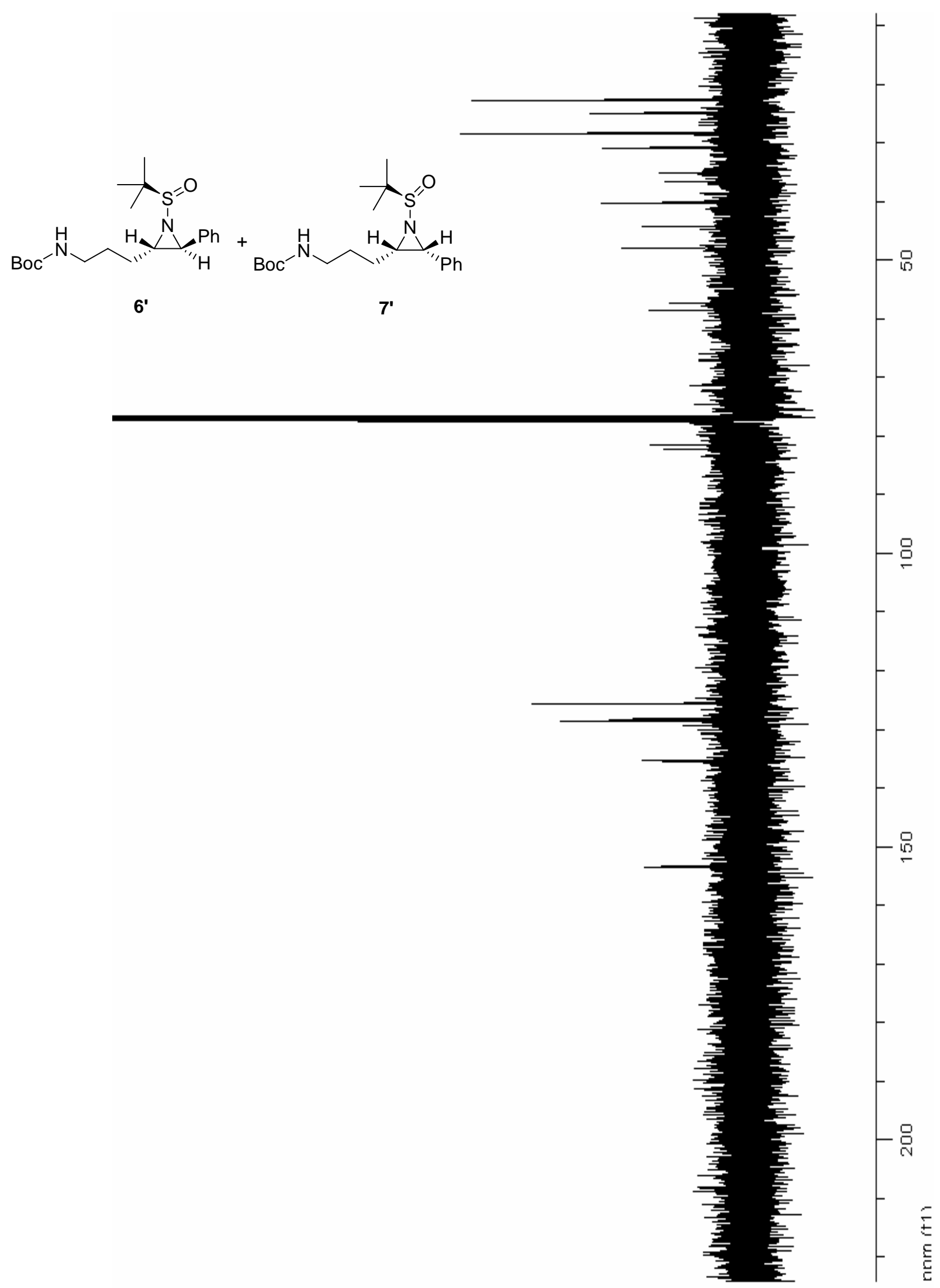


${ }^{1} \mathrm{H}$ NMR $\left(\mathrm{CDCl}_{3}\right)$ aziridines 13amajor:13aminor:14a

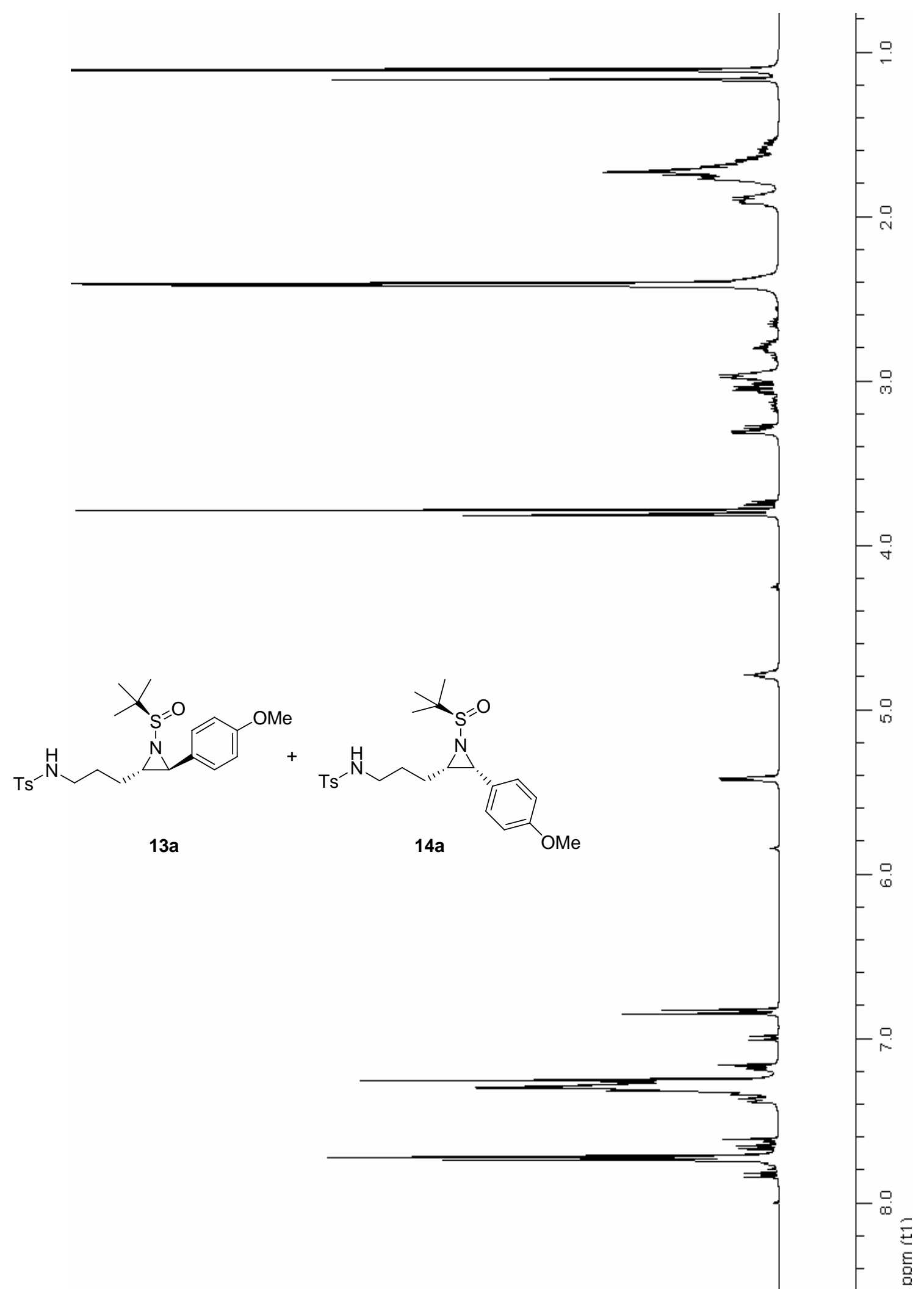


${ }^{13} \mathrm{C}$ NMR $\left(\mathrm{CDCl}_{3}\right)$ aziridines 13amajor:13aminor:14a

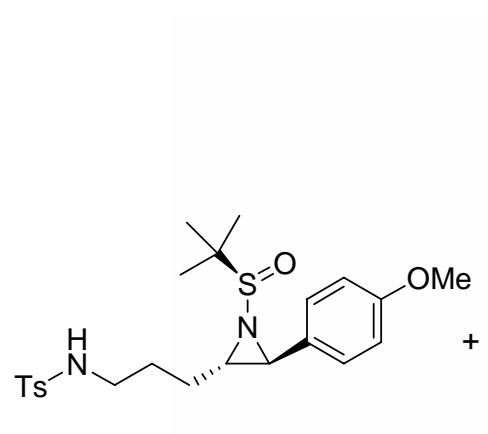

$13 a$

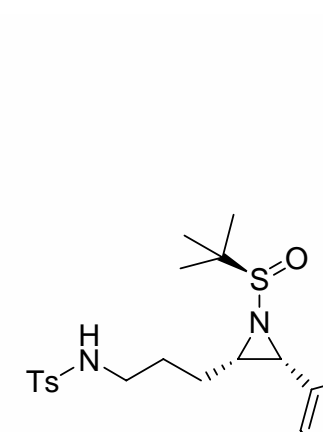

$14 a$
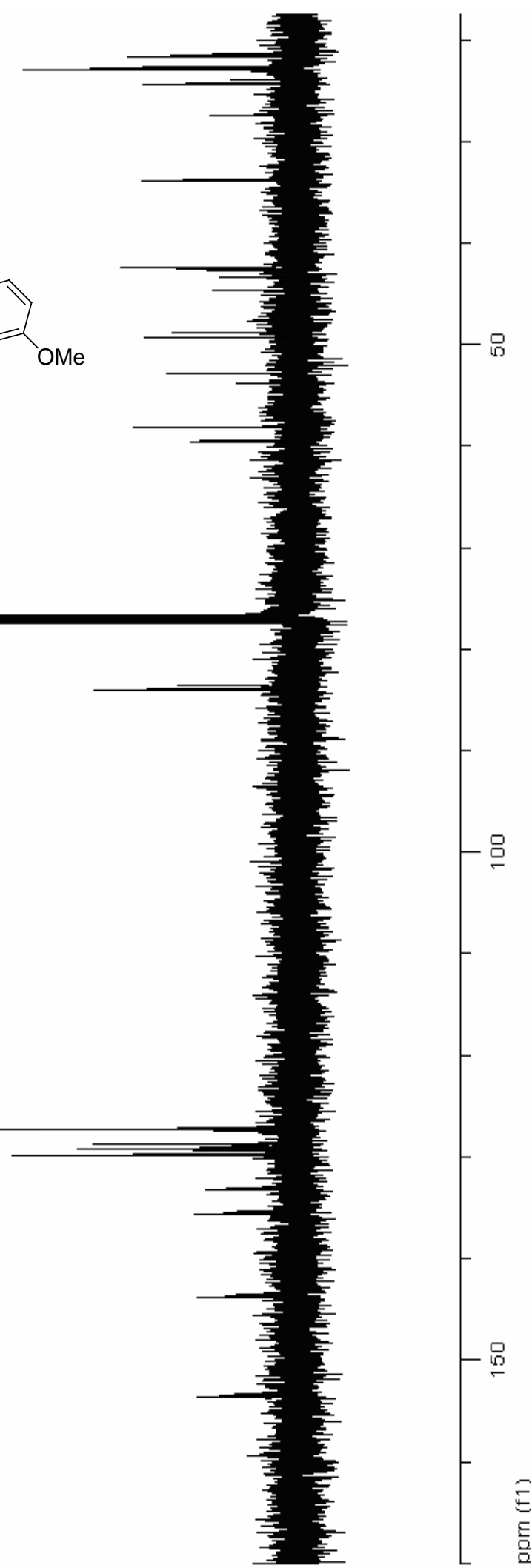
${ }^{1} \mathrm{H}$ NMR $\left(\mathrm{CDCl}_{3}\right)$ aziridines 13bminor:14b

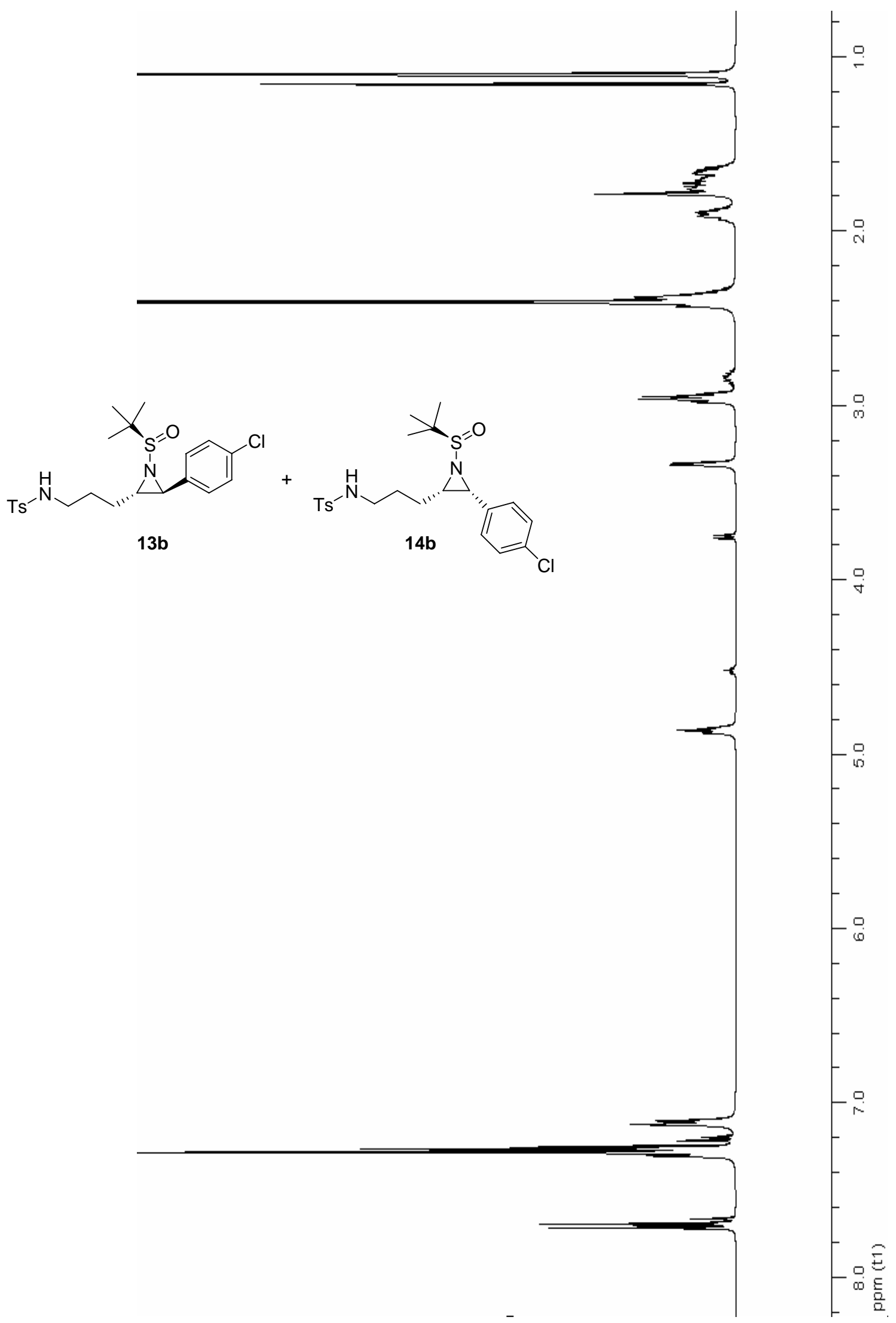




\section{${ }^{13} \mathrm{C}$ NMR $\left(\mathrm{CDCl}_{3}\right)$ aziridines 13bminor:14b}

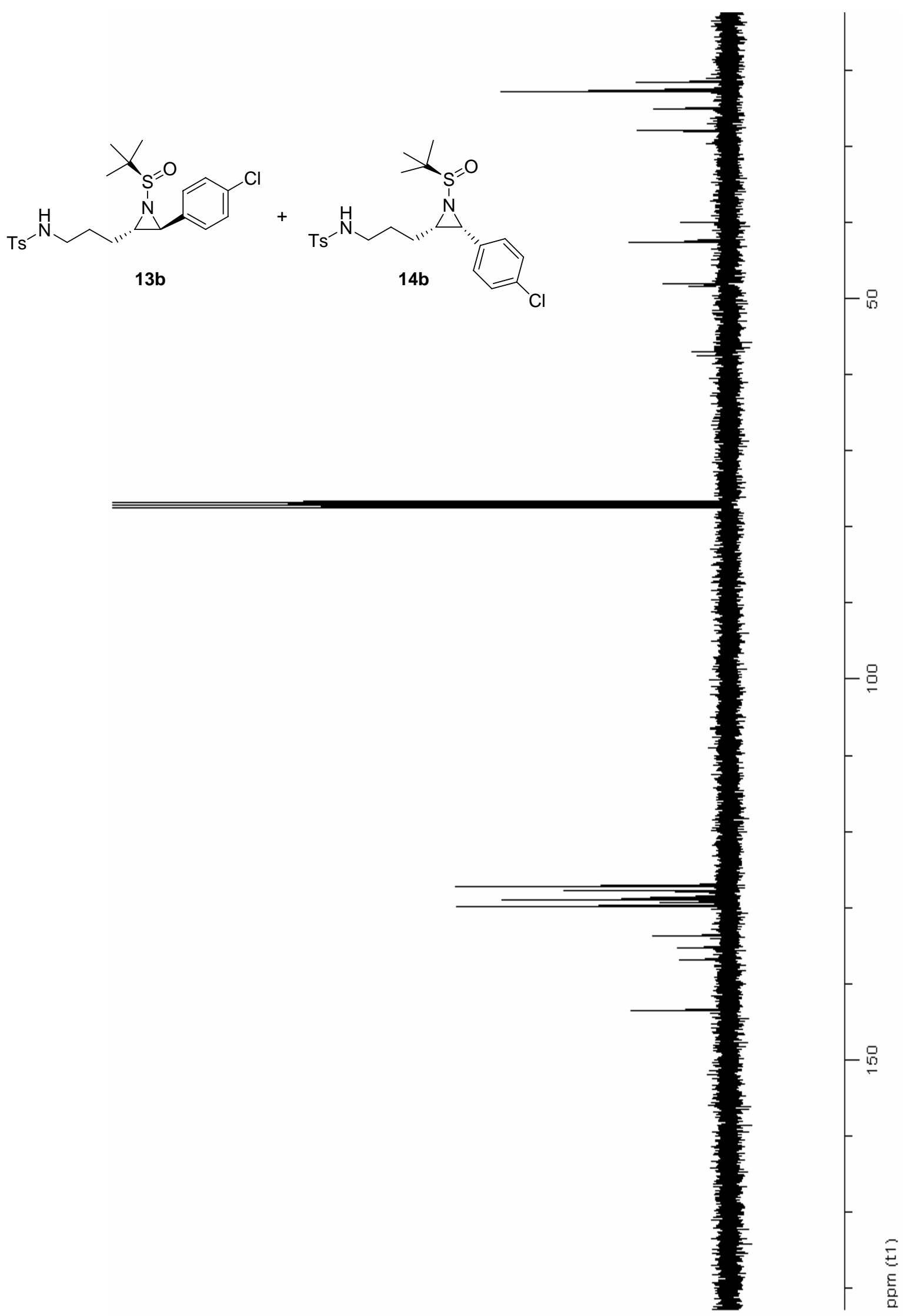


${ }^{1} \mathrm{H}$ NMR $\left(\mathrm{CDCl}_{3}\right)$ aziridine 13c

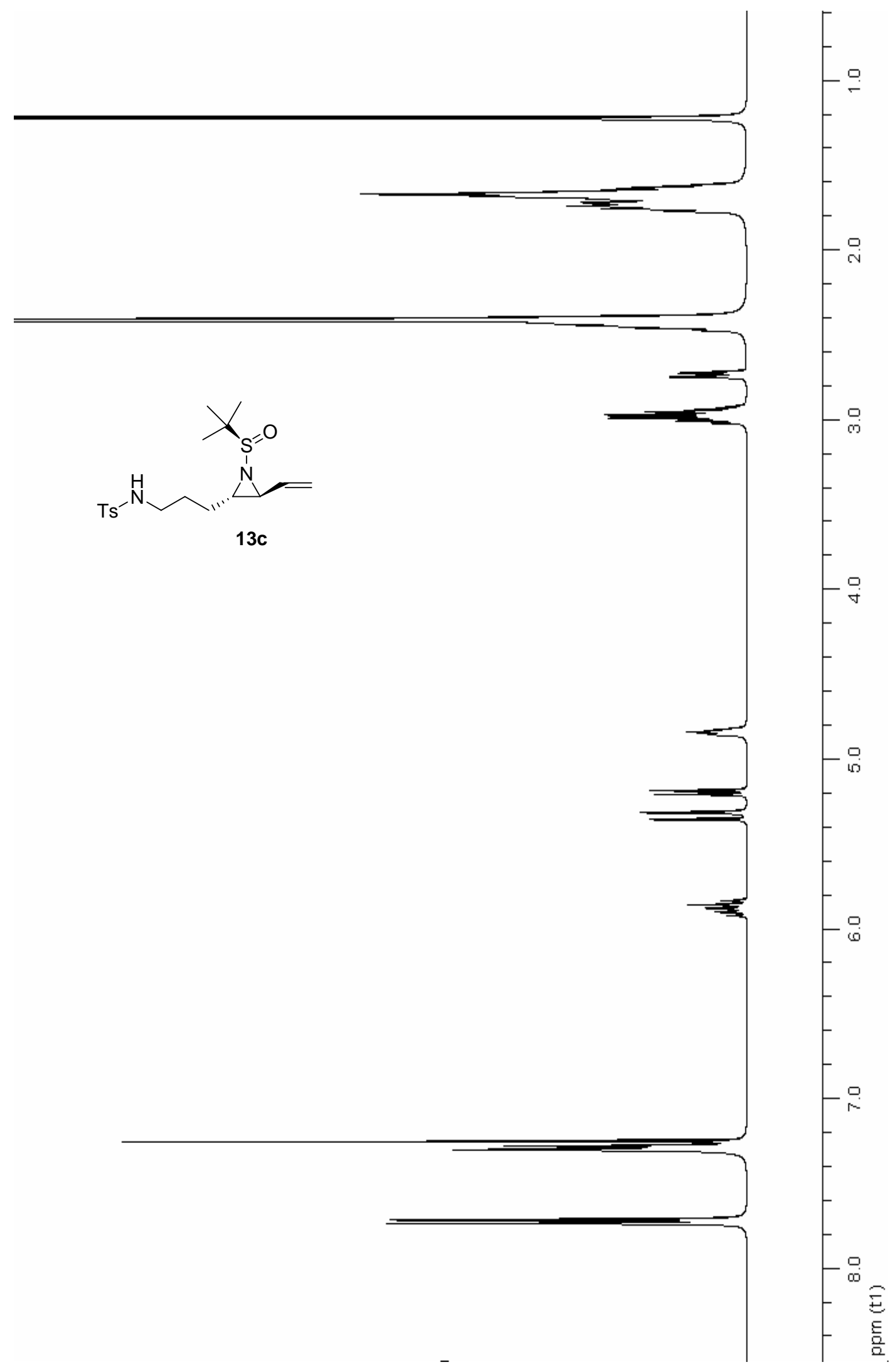




\section{${ }^{13} \mathrm{C}$ NMR $\left(\mathrm{CDCl}_{3}\right)$ aziridine 13c}
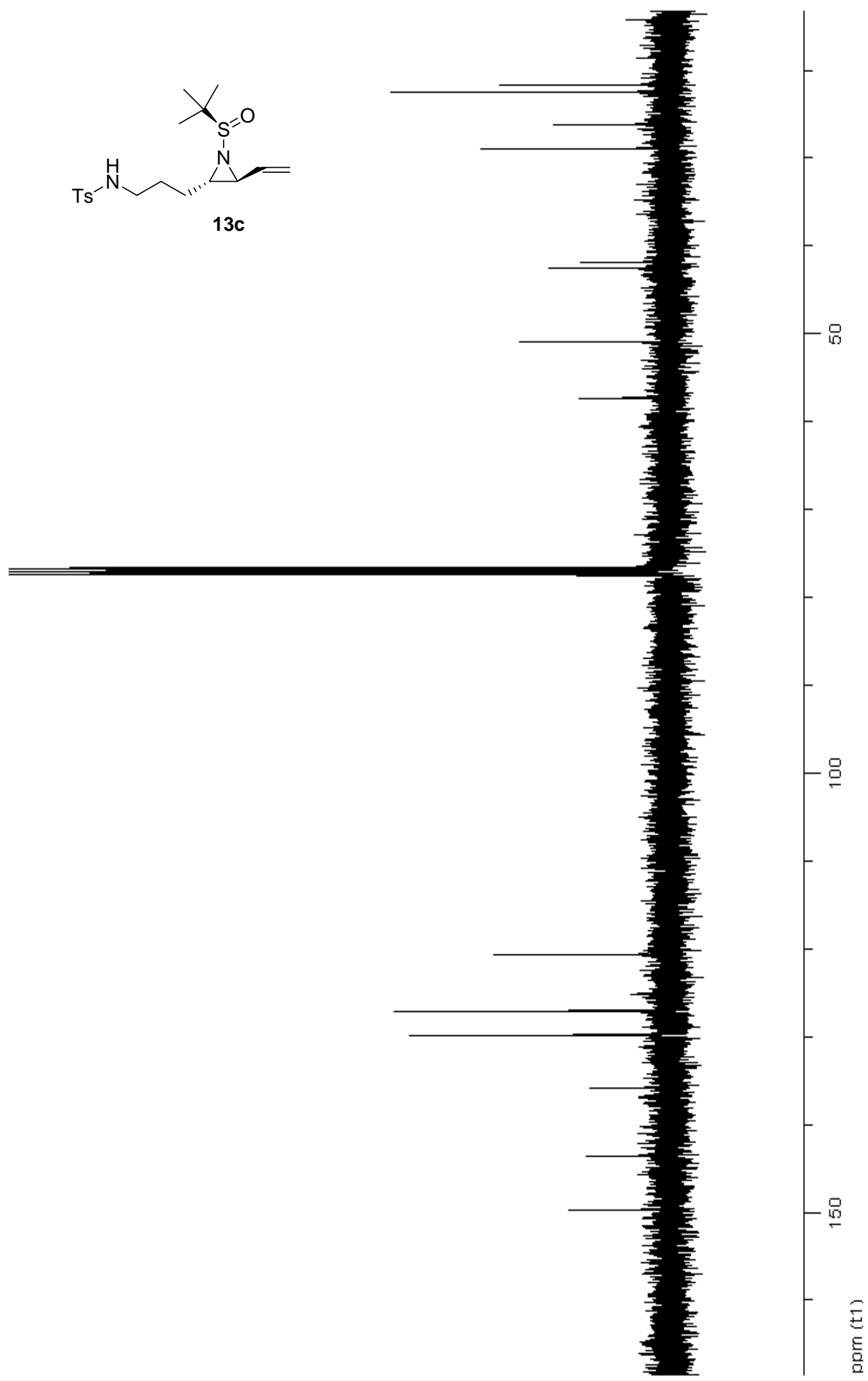
${ }^{1} \mathrm{H}$ NMR $\left(\mathrm{CDCl}_{3}\right)$ aziridine $14 \mathrm{c}$

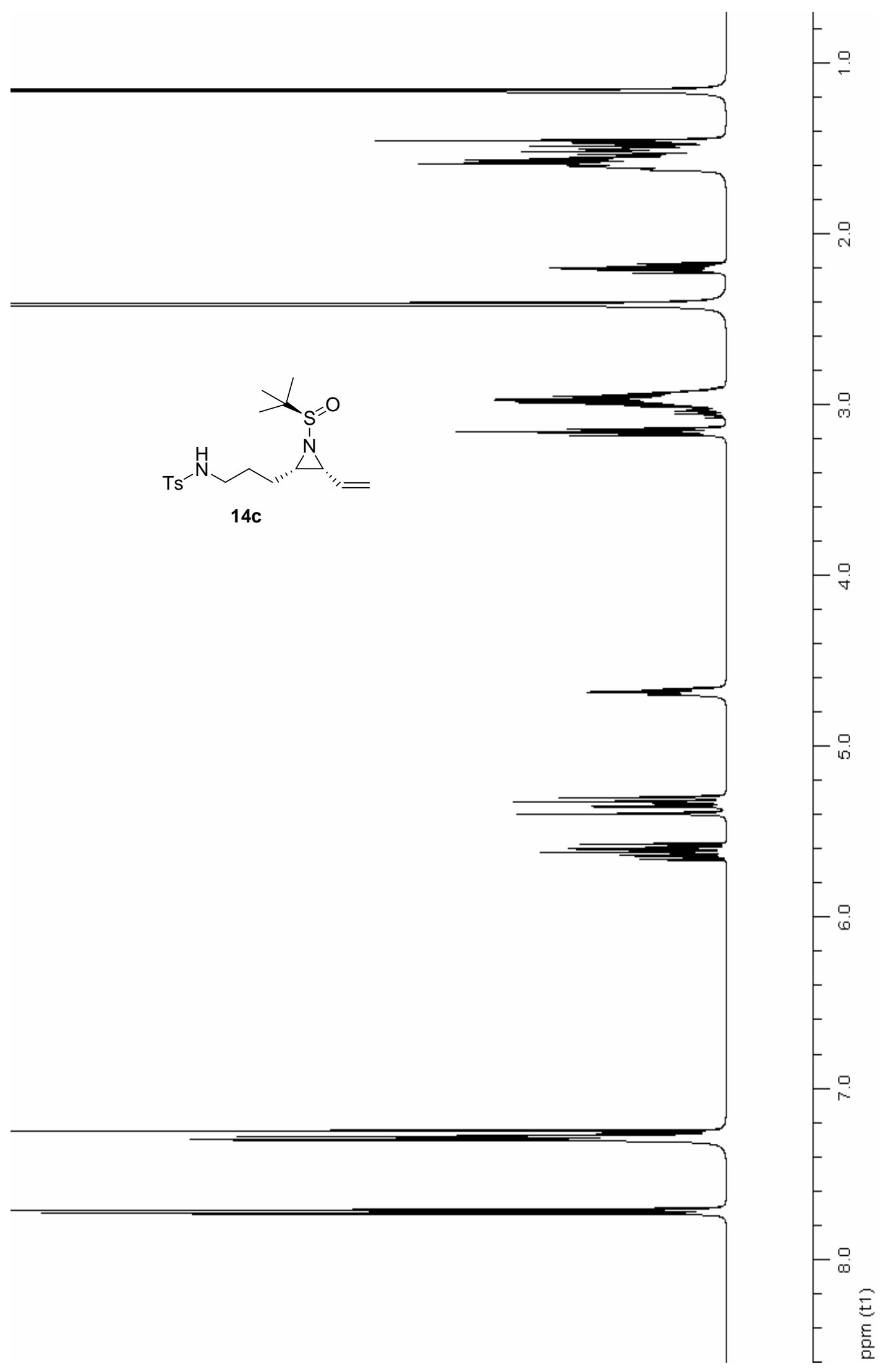


${ }^{13} \mathrm{C}$ NMR $\left(\mathrm{CDCl}_{3}\right)$ aziridine 14c

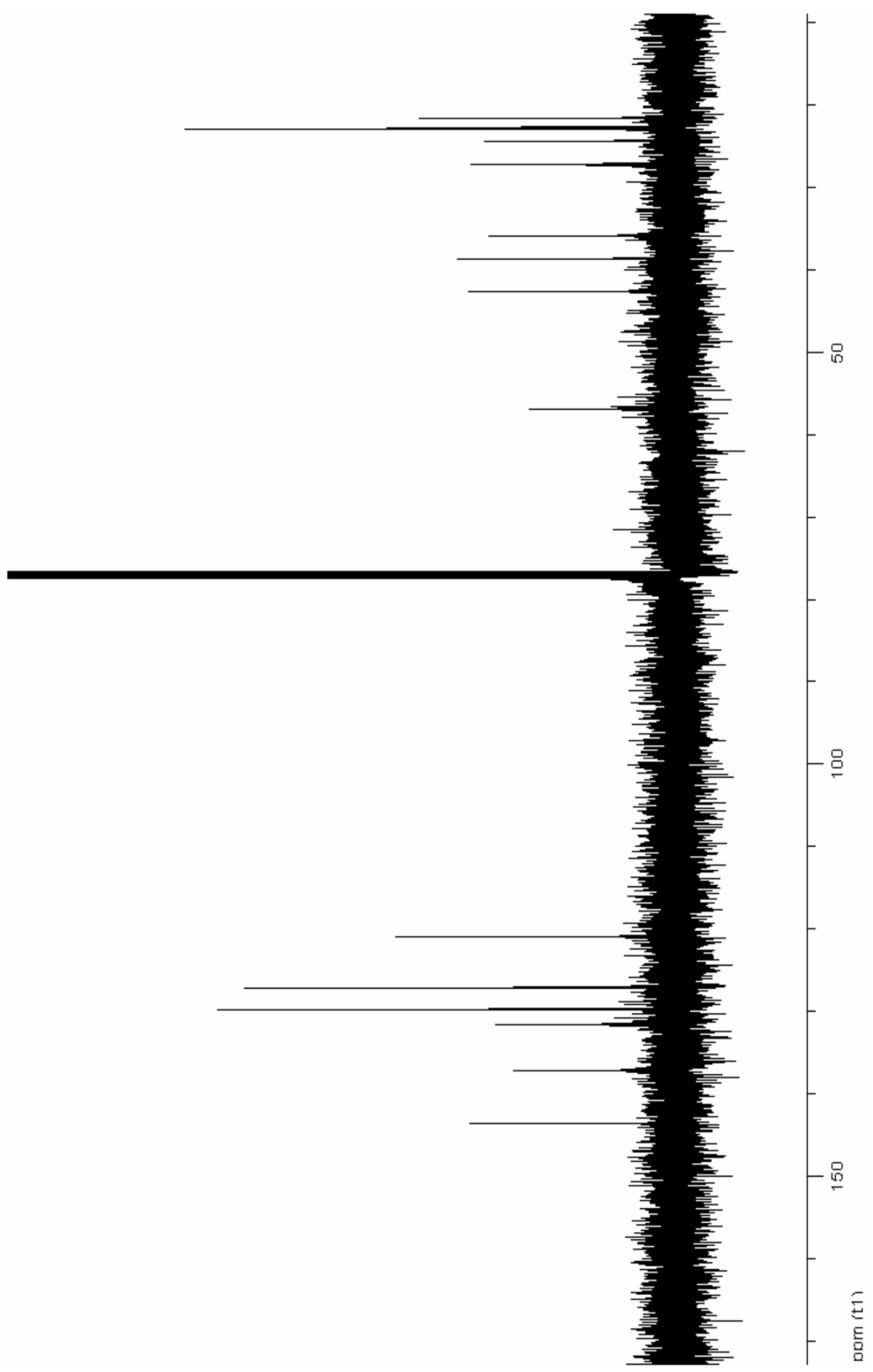


${ }^{1} \mathrm{H}$ NMR $\left(\mathrm{CDCl}_{3}\right)$ aziridines 13d:14d

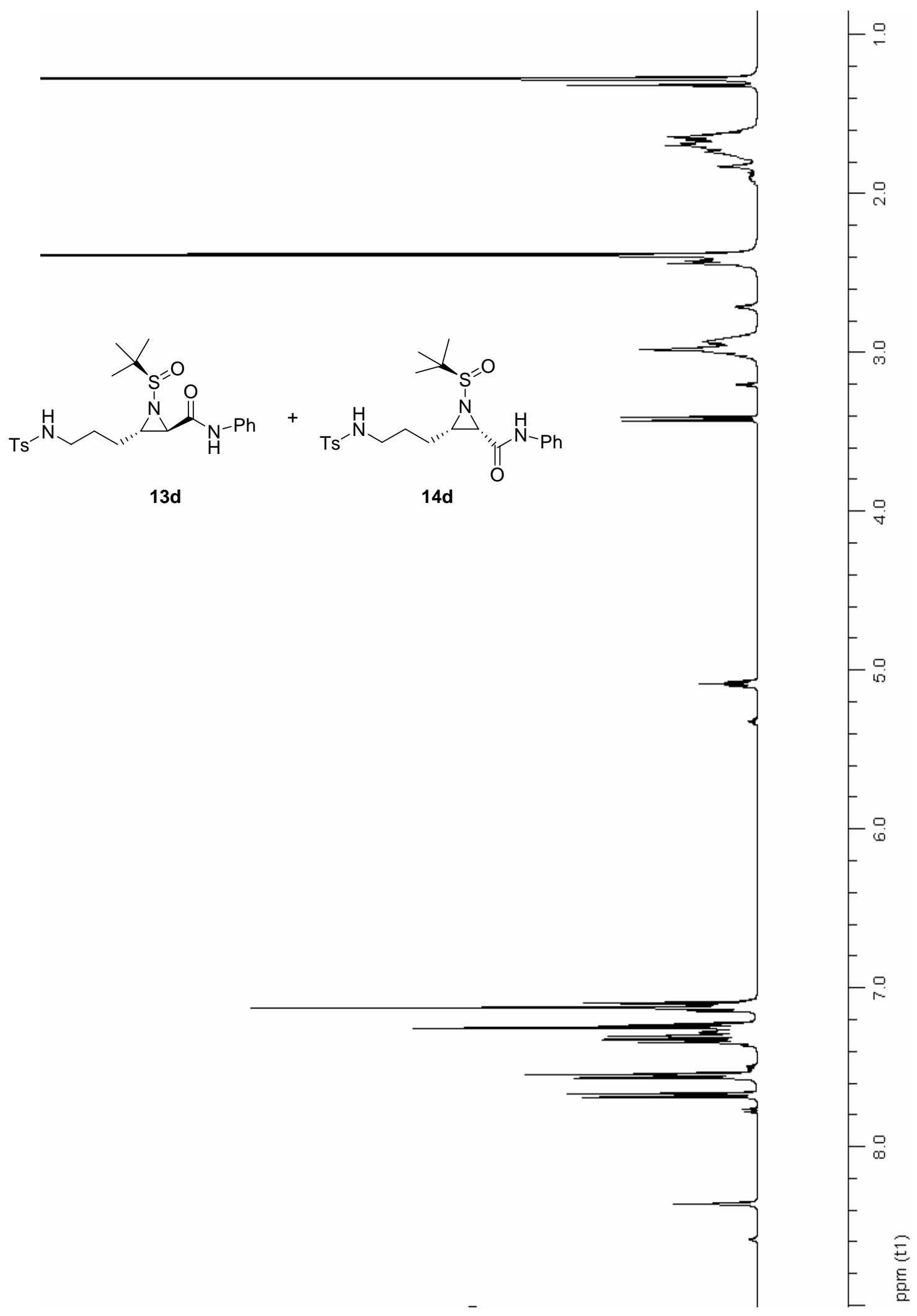


${ }^{13} \mathrm{C}$ NMR $\left(\mathrm{CDCl}_{3}\right)$ aziridines 13d:14d

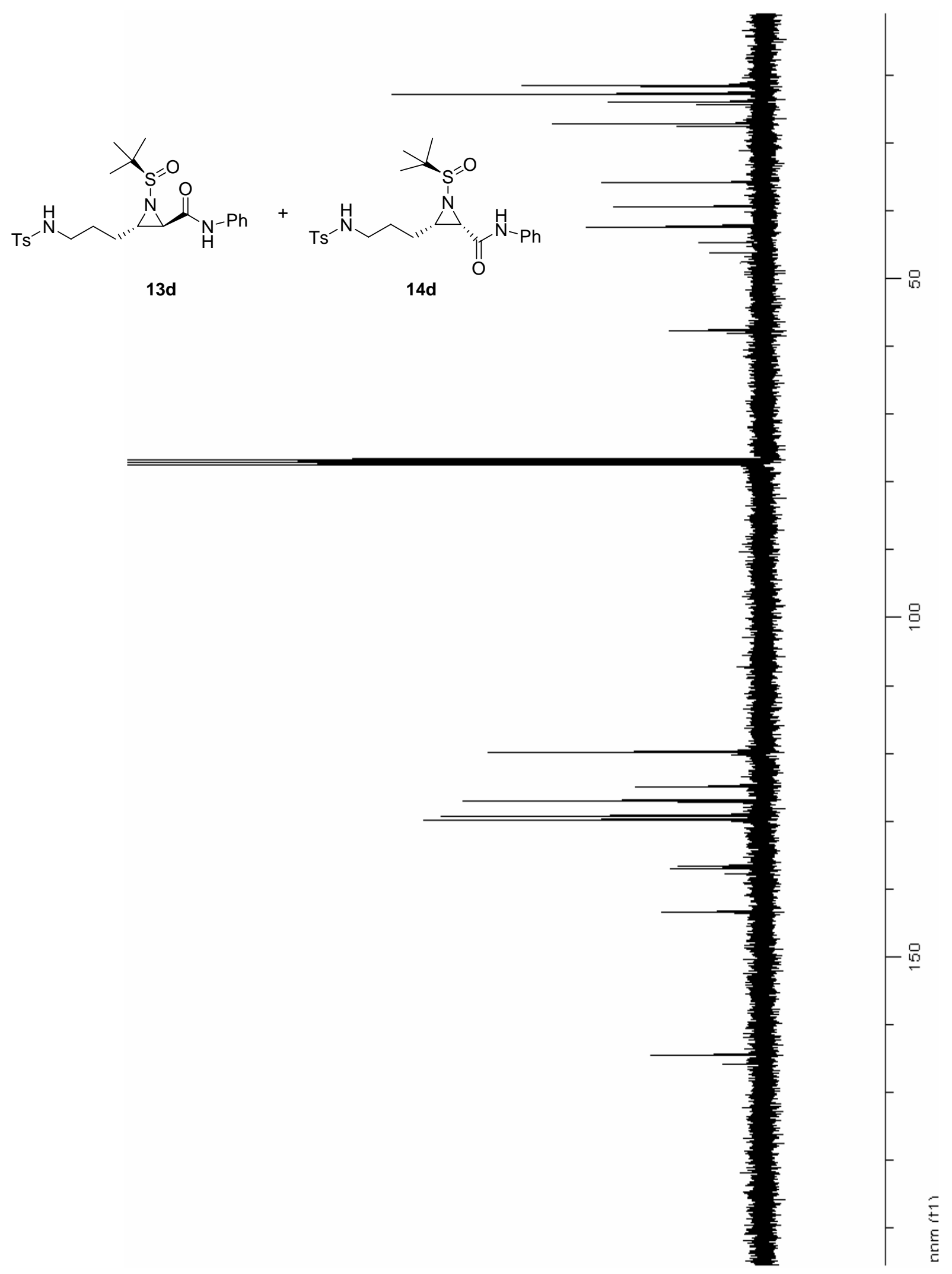


${ }^{1} \mathrm{H}$ NMR $\left(\mathrm{CDCl}_{3}\right)$ pyrrolidine 15

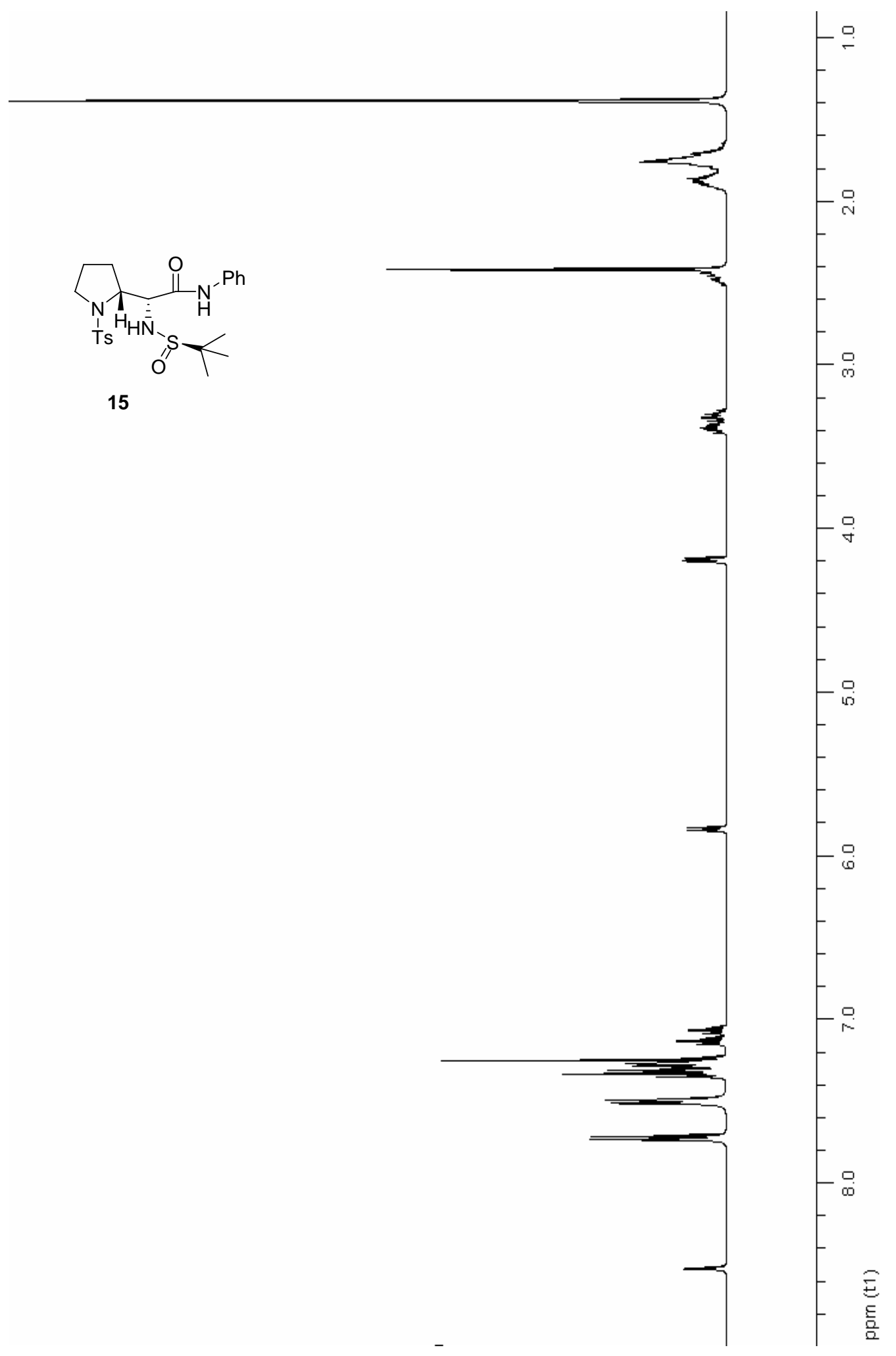




\section{${ }^{13} \mathrm{C}$ NMR $\left(\mathrm{CDCl}_{3}\right)$ pyrrolidine 15}
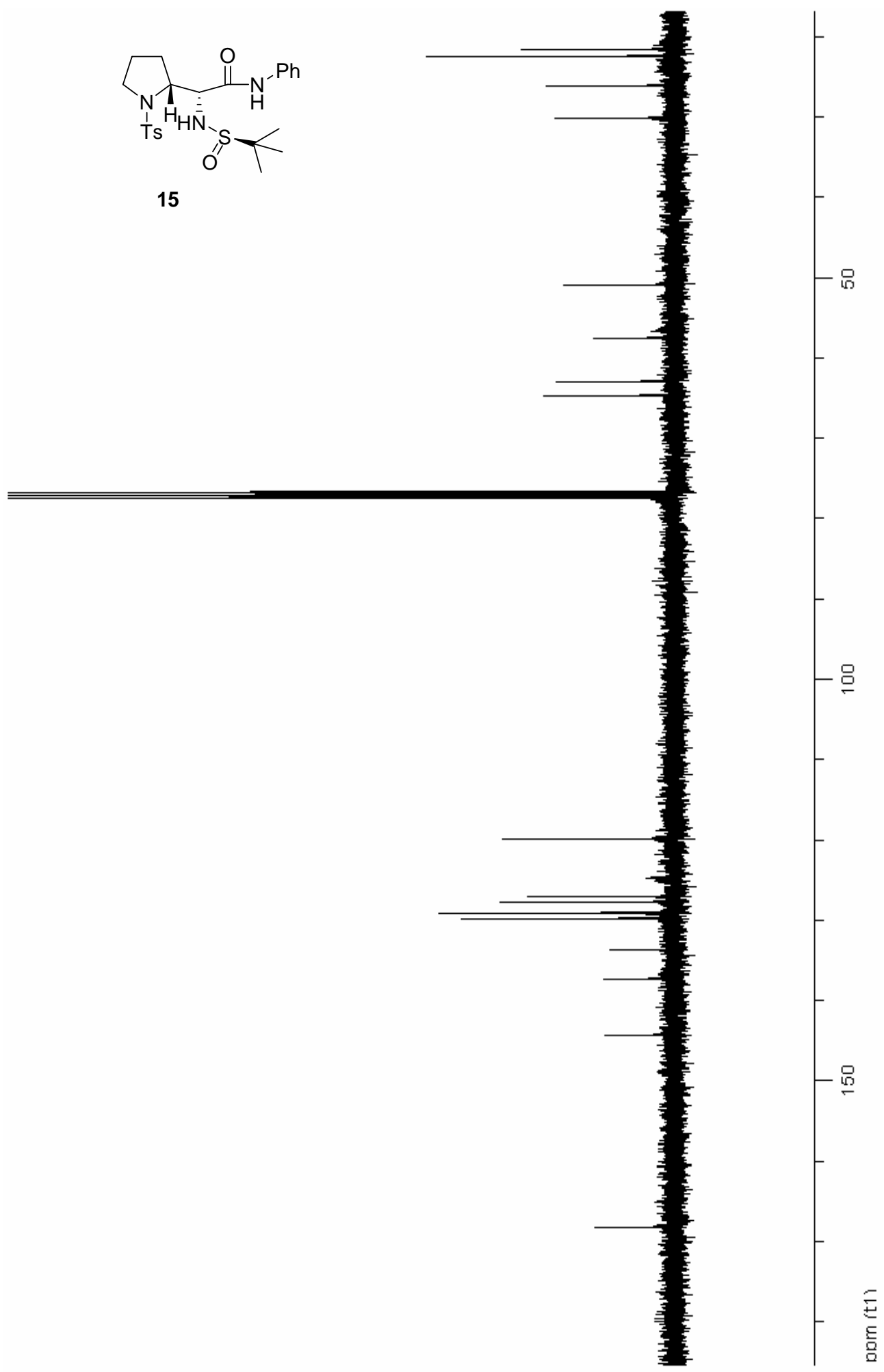
${ }^{1} \mathrm{H}$ NMR $\left(\mathrm{CDCl}_{3}\right)$ piperidine 16

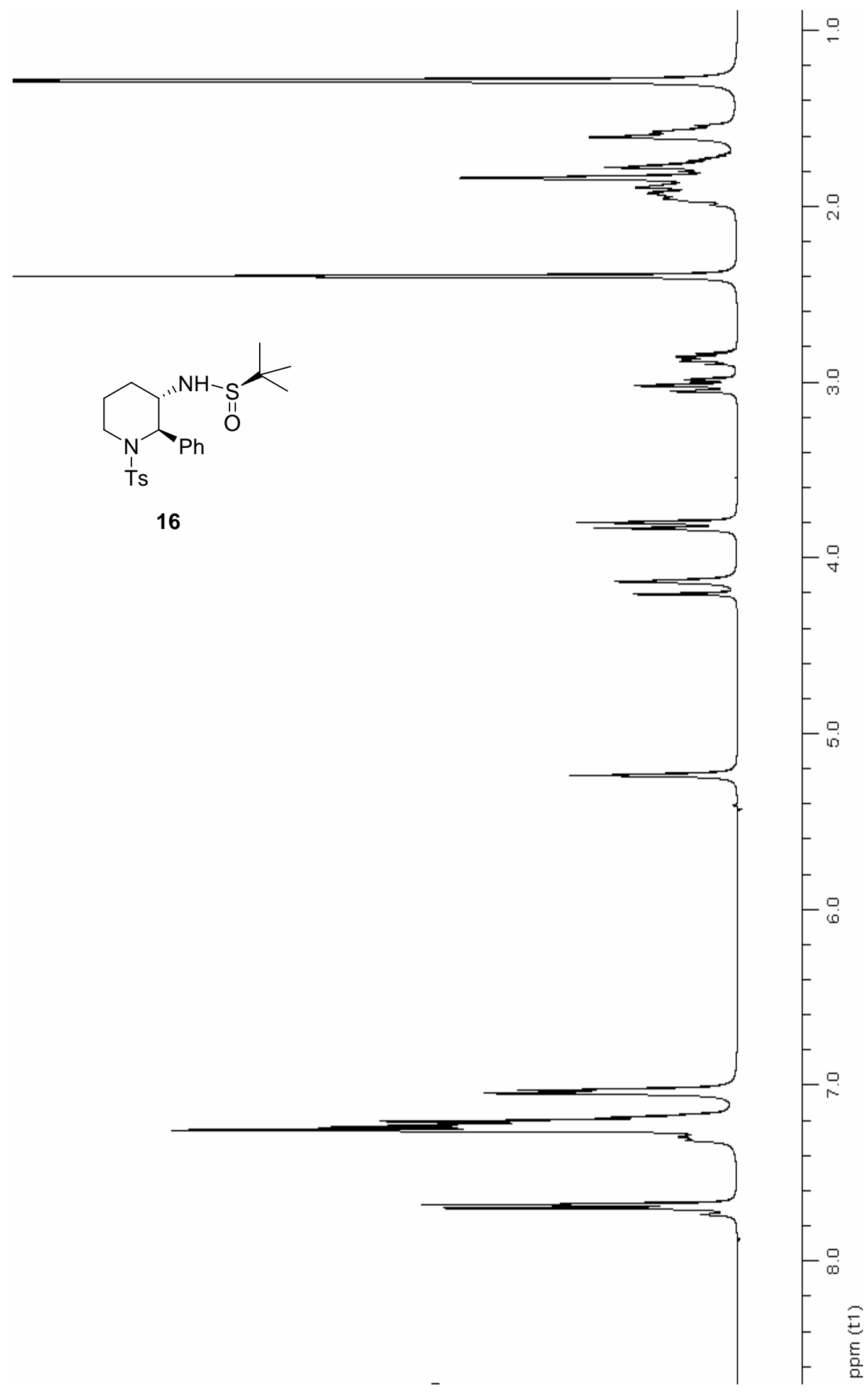


${ }^{13} \mathrm{C}$ NMR $\left(\mathrm{CDCl}_{3}\right)$ piperidine 16
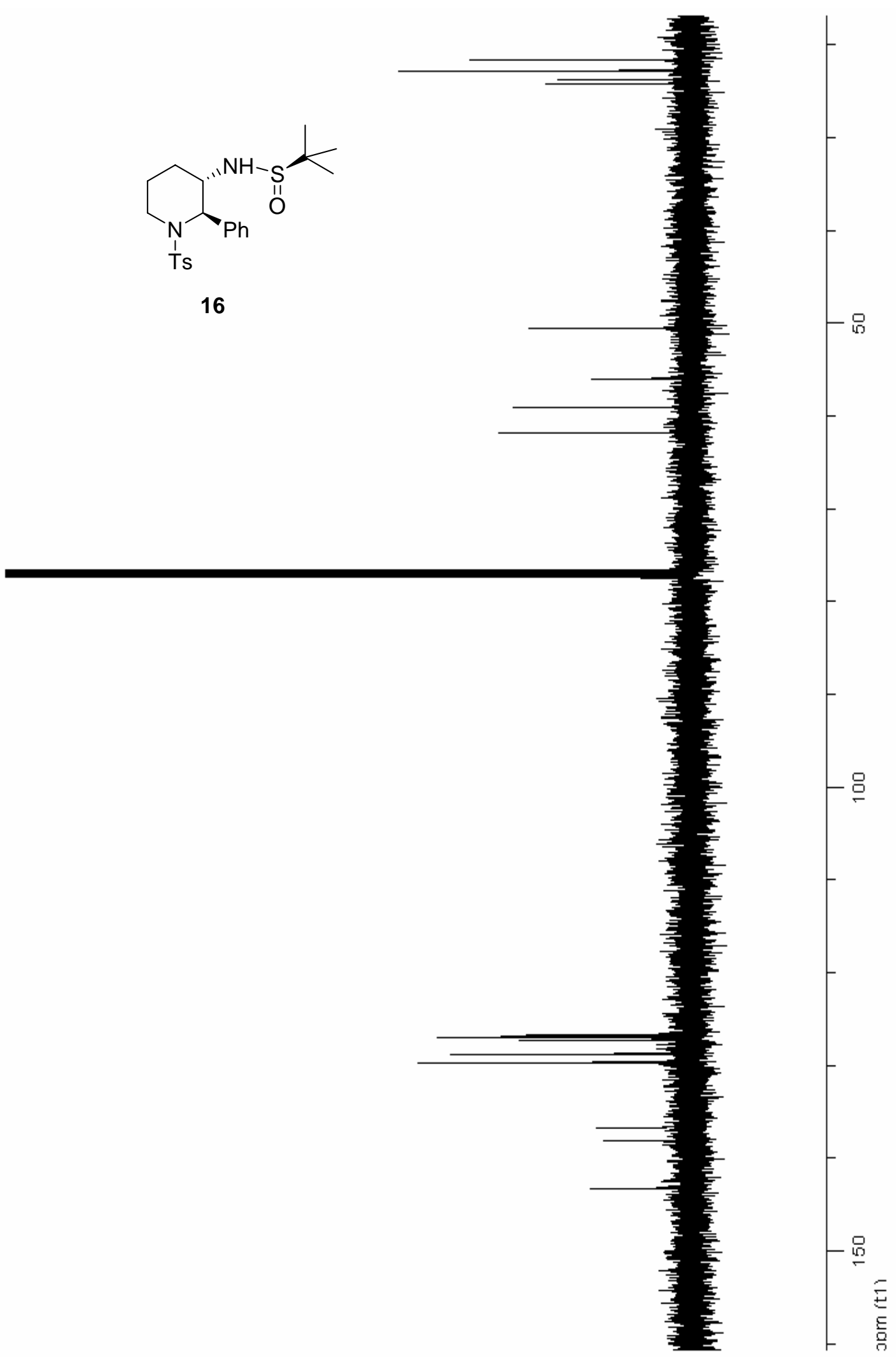
${ }^{1} \mathrm{H}$ NMR $\left(\mathrm{CDCl}_{3}\right)$ aziridine $\mathbf{1 7}$

Ts $-\mathrm{N}_{\text {(I")" }}^{\mathrm{H}}$
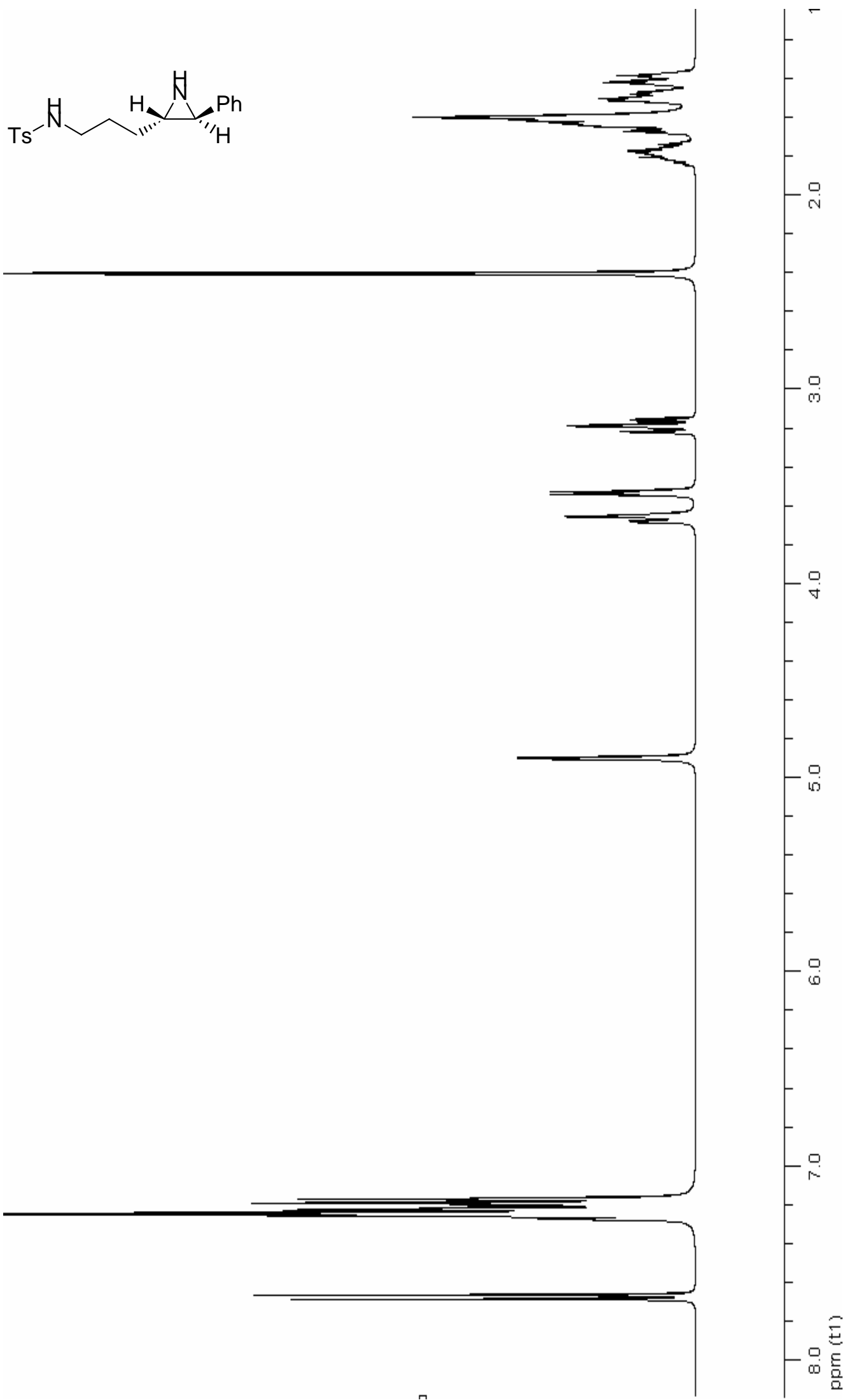
${ }^{13} \mathrm{C}$ NMR $\left(\mathrm{CDCl}_{3}\right)$ aziridine 17
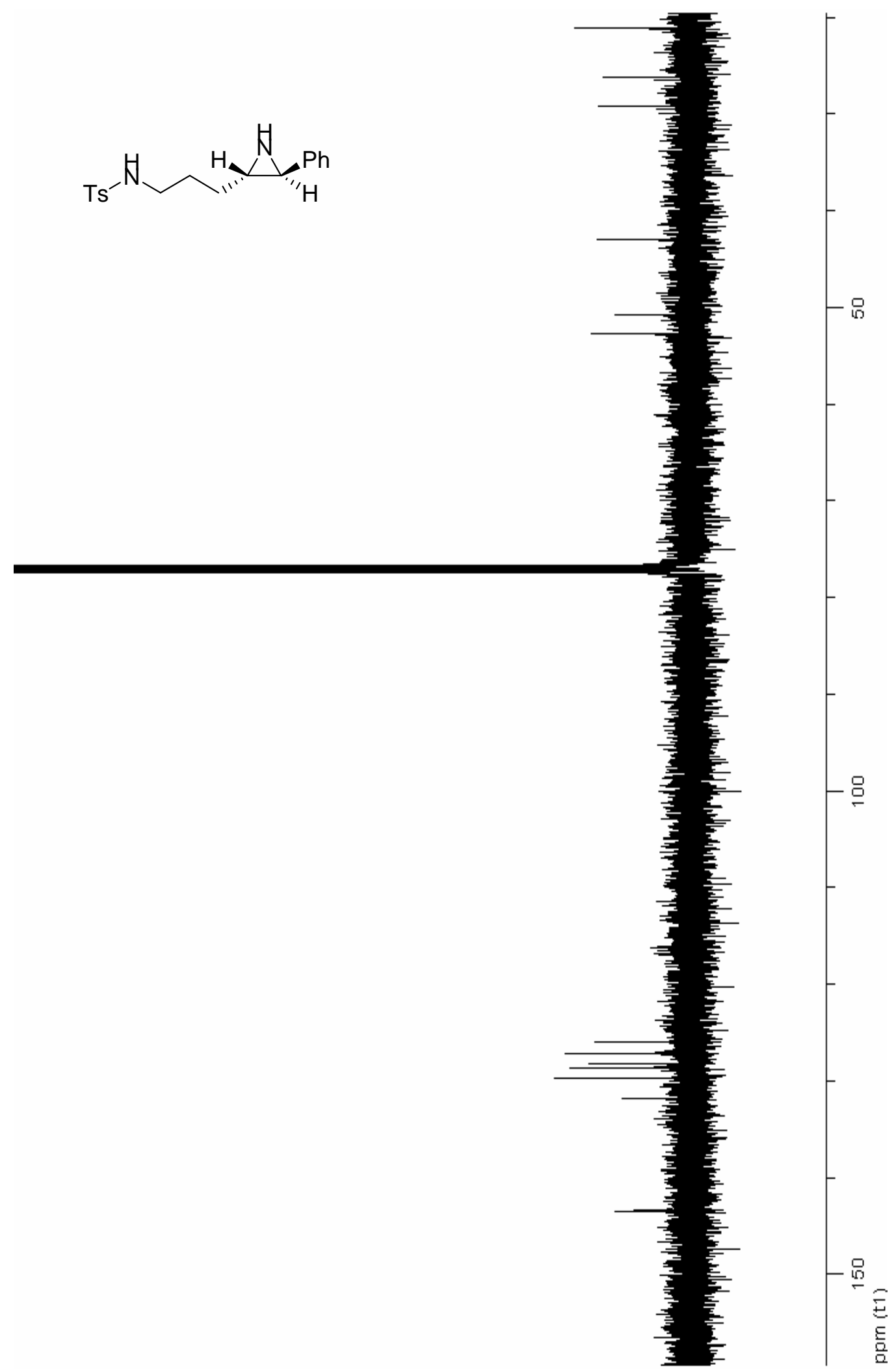


\section{References}

(1) Unthank, M. G.; Husain, N.; Aggarwal, V. K. Angew. Chem. Int. Ed., 2006, 45, 7066. 